\title{
Phase speed and attenuation in bubbly liquids inferred from impedance measurements near the individual bubble resonance frequency
}

\author{
Preston S. Wilson, ${ }^{\text {a) }}$ Ronald A. Roy, and William M. Carey \\ Department of Aerospace and Mechanical Engineering, Boston University, 110 Cummington Street, \\ Room 101, Boston, Massachusetts 02215
}

(Received 30 July 2004; revised 21 December 2004; accepted 22 December 2004)

\begin{abstract}
In the ocean, natural and artificial processes generate clouds of bubbles that scatter and attenuate sound. Measurements have shown that at the individual bubble resonance frequency, sound propagation in this medium is highly attenuated and dispersive. The existing theory to explain this behavior is deemed adequate away from resonance. However, due to excessive attenuation near resonance, little experimental data exists for a comparison with model predictions. An impedance tube was developed specifically for exploring this regime. The effective medium phase speed and attenuation were inferred from measurements of the surface impedance of a layer of bubbly liquid composed of air bubbles and distilled water, for void fractions from $6.2 \times 10^{-5}$ to $5.4 \times 10^{-4}$ and bubble sizes centered around $0.62 \mathrm{~mm}$ in radius. Improved measurement speed, accuracy, and precision is possible with the new instrument, and both instantaneous and time-averaged measurements were obtained. The phase speed and attenuation at resonance was observed to be sensitive to the bubble population statistics and agreed with an existing model [J. Acoust. Soc. Am. 85, 732-746 (1989)], within the uncertainty of the bubble population parameters. Agreement between the model and the data reported here is better than for the data that was available when the model was originally published. (C) 2005 Acoustical Society of America.
\end{abstract}

[DOI: $10.1121 / 1.1859091]$

PACS numbers: 43.30.Es, 43.35.Bf [SLB]

Pages: $1895-1910$

\section{INTRODUCTION}

The acoustics of bubbly liquids is important to many areas of study, including acoustical oceanography, shallow water sonar, ship wakes, air/sea interfacial mass transfer, medical ultrasound, and nuclear reactor technology. ${ }^{1,2}$ Sound speed dispersion and attenuation in clean suspensions of bubbles is well understood when the acoustic excitation frequency is well below the resonance frequency of any bubble and the volume fraction of air is below a few percent. The volume fraction $(\mathrm{VF})$ of air, or void fraction $\beta$, is defined as

$$
\beta=V_{g} / V_{m},
$$

where $V_{g}$ is the volume of the gas phase and $V_{m}$ is the total volume of the mixture.

As the excitation frequency approaches the range of bubble resonance frequencies, the dispersion and attenuation grows rapidly. This effect is amplified further for bubble size distributions with sharp peaks. Commander and Prosperetti ${ }^{3}$ compared their propagation model to the experimental data available at the time and found poor agreement in such cases, even for void fractions as low as $3.7 \times 10^{-4}$. This has been attributed to the effects of multiple scattering between neighboring bubbles ${ }^{3}$ and experimental difficulties in measuring acoustic properties in the presence of high attenuation. ${ }^{4} \mathrm{Re}-$ cently, a number of authors ${ }^{5-8}$ have put forward models that address multiple scattering. Unfortunately, experimental un-

\footnotetext{
${ }^{a}$ Current affiliation: University of Texas at Austin, Department of Mechanical Engineering; electronic mail: pswilson@mail.utexas.edu
}

certainty in the data that exists, and the absence of data for much of the parameter space, prevents an adequate evaluation of competing models. ${ }^{3,9}$ The fact that the data obtained by Silberman in 1957 is the most commonly cited data further emphasizes this situation.

There are two reasons for the scarcity of reliable experimental data for cases where resonance is important. (1) The attenuation, which is of $O(10) \mathrm{dB} / \mathrm{cm}$ and higher, prevents the use of traditional standing wave techniques. Even pulse propagation techniques fail because sound transmission beyond the near field of the source is not readily achieved. (2) Propagation in bubbly liquids is very sensitive to the void fraction, and to the bubble size and spatial distributions. A sufficiently accurate experimental determination and control of these parameters is difficult.

An approach designed to overcome these difficulties is described in the present work. In order to deal with the problem of high attenuation, measurement directly within the highly attenuating region of bubbly liquid was avoided. Instead, the frequency-dependent, complex impedance $z_{s}$ at the surface of a layer of bubbly liquid was measured using a modified version of the standard two-sensor impedance tube. ${ }^{10}$ This was accomplished by measuring the acoustic pressures at two known positions within the bubble-free section of the impedance tube. Equations of plane wave acoustics ${ }^{10,11}$ were used to relate these acoustic pressures to the impedance at the interface between the bubble-free liquid and the bubbly liquid. This impedance is equivalent to the acoustic pressure/velocity ratio that would exist just inside the bubbly liquid if it were a homogeneous fluid. In the 
present case, this measured impedance is equivalent to the effective impedance of the bubbly liquid and the effective propagation parameters are inferred from the measured impedance $z_{s}$. For the level of attenuation encountered in the present work, only plane progressive waves cross the measurement surface; therefore $z_{s}$ is equivalent to the specific acoustic impedance $z=\rho c$. Since $z$ is measured and the effective mixture density $\rho$ is known, the phase speed and attenuation can be calculated from the complex sound speed $c$. Measurements of the phase speed and attenuation obtained in this way for a bubbly liquid composed of air and distilled water are presented. Void fractions ranging from $6.2 \times 10^{-5}$ to $5.4 \times 10^{-4}$ and bubble sizes centered around $0.62 \mathrm{~mm}$ in radius were investigated.

These measurements were compared with the model put forth by Commander and Prosperetti. ${ }^{3}$ The sensitivity of the model to input parameters is discussed in Sec. II. A description of the experiment is given in Sec. III. The results of a time-averaged experiment are discussed in Sec. IV. Finally, the results of a time-resolved experiment, used to investigate the effects of bubble population variability, are discussed in Sec. V.

\section{OVERVIEW OF PROPAGATION MODEL}

In 1989, Commander and Prosperetti ${ }^{3}$ brought together previously published results and composed a model for sound propagation in bubbly liquid which accounted for energy dissipation in a detailed way. Their final result is shown below for reference. Consider a host liquid with sound speed $c_{l}$, density $\rho_{l}$, viscosity $\mu$, surface tension $\sigma$, and equilibrium pressure $P_{\infty}$. This liquid contains bubbles composed of a gas with thermal diffusivity $D_{g}$ and ratio of specific heats $\gamma$. The bubbly liquid is treated like a homogeneous mixture in which the complex mixture sound speed $c_{m}$ is given by

$$
\frac{c_{l}^{2}}{c_{m}^{2}}=1+4 \pi c_{l}^{2} \int_{0}^{\infty} \frac{a \wp(a) d a}{\omega_{0}^{2}-\omega^{2}+2 i b \omega},
$$

where $\omega$ is the circular excitation frequency and $\wp(a) d a$ is the number of bubbles per unit volume with equilibrium radius between $a$ and $a+d a$. The damping coefficient for a bubble of radius $a$ driven at frequency $\omega$ is given by

$$
b=\frac{2 \mu}{\rho_{l} a^{2}}+\frac{P_{\mathrm{b}, \mathrm{e}}}{2 \rho_{l} a^{2} \omega} \operatorname{Im} \Phi+\frac{\omega^{2} a}{2 c_{l}},
$$

where the three terms are due to viscous, thermal, and acoustic dissipation effects, respectively and $\operatorname{Im} \Phi$ is the imaginary part of

$$
\Phi=\frac{3 \gamma}{1-3(\gamma-1) i X\left[(i / X)^{1 / 2} \operatorname{coth}(i / X)^{1 / 2}-1\right]} .
$$

The bubble resonance frequency is

$$
\omega_{0}^{2}=\frac{P_{\mathrm{b}, \mathrm{e}}}{\rho_{l} a^{2}}\left(\operatorname{Re} \Phi-\frac{2 \sigma}{a P_{\mathrm{b}, \mathrm{e}}}\right),
$$

where $\operatorname{Re} \Phi$ is the real part of $\Phi$. The definition,

$$
X=D_{g} / \omega a^{2},
$$

TABLE I. Values of the physical parameters used for evaluation of Eq. (2).

\begin{tabular}{lc}
\hline \hline$c_{l}=1481 \mathrm{~m} / \mathrm{s}$ & $D_{g}=2.08 \times 10^{-5} \mathrm{~m}^{2} / \mathrm{s}$ \\
$\rho_{l}=998 \mathrm{~kg} / \mathrm{m}^{3}$ & $P_{\infty}=101.3 \mathrm{kPa}$ \\
$\sigma=0.0725 \mathrm{~N} / \mathrm{m}$ & $\mu=0.001 \mathrm{~Pa} \mathrm{~s}$ \\
$\gamma=1.4$ & \\
\hline \hline
\end{tabular}

has been used and the quantity $P_{\mathrm{b}, \mathrm{e}}$ represents the equilibrium pressure in the bubble,

$$
P_{\mathrm{b}, \mathrm{e}}=P_{\infty}+\frac{2 \sigma}{a}
$$

where $P_{\infty}$ is the hydrostatic pressure in the liquid. The wave number for propagation within the bubbly mixture can then be written from Eq. (2) as

$$
k_{m}^{2}=\frac{\omega^{2}}{c_{l}^{2}}+4 \pi \omega^{2} \int_{0}^{\infty} \frac{a \wp(a) d a}{\omega_{0}^{2}-\omega^{2}+2 i b \omega} .
$$

\section{A. Sensitivity to small changes in bubble size}

Small changes in bubble population parameters (or small uncertainties in their experimental determination) can have large effects on the (predicted) phase speed and attenuation in bubbly fluids. This will be demonstrated for different bubble size distributions using Eq. (2), assuming air bubbles in pure water at $20^{\circ} \mathrm{C}$ and the corresponding physical parameters given in Table I.

Consider a monodisperse bubble population, given by

$$
\wp(a)=n \delta(a-\bar{a}),
$$

where $n$ is the number of bubbles per unit volume with equilibrium radius $\bar{a}$ and the void fraction is

$$
\beta=\frac{4}{3} \pi \bar{a}^{3} n
$$

Substitution into Eq. (2) yields

$$
\frac{1}{c_{m}^{2}}=\frac{1}{c_{l}^{2}}+\frac{4 \pi \bar{a} n}{\omega_{0}^{2}-\omega^{2}+2 i b \omega} .
$$

Phase speed $V$ and attenuation coefficient $A$ were calculated using Eq. (11) for void fraction $\beta=10^{-4}$ and three bubble radii, $\bar{a}=0.558,0.62$, and $0.682 \mathrm{~mm}$. Both the phase speed and the attenuation curves, shown in Fig. 1, shift along the frequency axis as bubble size is varied. A small increase in peak attenuation is seen as bubble size decreases and the minimum phase speed increases slightly. The latter effect is not prominent on the logarithmically scaled axis, but is indeed present. The behavior of the attenuation for these parameters is governed by thermal dissipation, which is inversely proportional to the bubble radius squared.

Note that the pronounced effects shown in Fig. 1 are induced by only a $10 \%$ variation in bubble radius, or specifically, $\pm 62 \mu \mathrm{m}$. The experimental ability to discern the difference between acoustic signals of 5.3 and $5.8 \mathrm{kHz}$ (the frequencies of peak attenuation for the two smaller bubble sizes shown in Fig. 1) is often greater than our ability to discern the difference in the two corresponding bubble populations. If one were only interested in the size of a few 

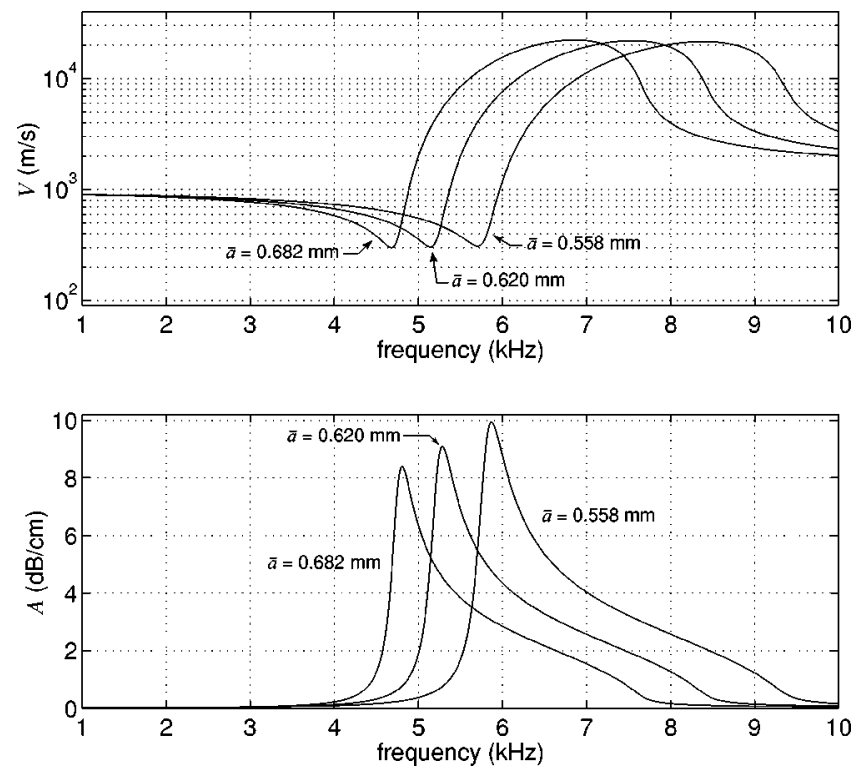

FIG. 1. The effect of the bubble size is demonstrated. Phase speed (upper) and attenuation (lower) is shown for void fraction $\beta=10^{-4}$ and three bubble radii, which represent a $\pm 10 \%$ change in radius.

bubbles this would not be the case; high magnification optical methods would provide sufficient resolution. However, when one endeavors to measure the sizes of hundreds of bubbles in motion, the statement bears a much greater significance.

\section{B. Sensitivity to small changes in bubble size distribution width}

The behavior of continuous bubble size distributions will now be considered. Foreshadowing what will be discussed in the experimental part of this work, the normal distribution will be explored. The probability density function of a normally distributed random variable is defined by

$$
\wp(a)=\frac{C}{s \sqrt{2 \pi}} \exp \left[-\frac{\left(a-a_{0}\right)^{2}}{2 s^{2}}\right],
$$

where $a_{0}$ and $s$ represent the mean value and the standard deviation of the distribution, respectively. Here, $a_{0}$ is taken to be the mean bubble radius, and $C$ is used as a scaling parameter such that the void fraction $\beta$ is given by

$$
\beta=\frac{4 \pi}{3} \int_{a_{\min }}^{a_{\max }} \wp(a) a^{3} d a .
$$

The purpose is to isolate the effect of increasing the standard deviation of the distribution, that is, the effect of widening the distribution.

Three distribution widths are considered, $s=0.02,0.03$, and $0.04 \mathrm{~mm}$, along with the following parameters: $\beta$ $=10^{-4}$, and $a_{\min }=0.50, a_{0}=0.62, a_{\max }=0.75 \mathrm{~mm}$. With $\wp(a)$ thus described, Eq. (2) was evaluated numerically using an adaptive Simpson quadrature technique. The results are shown in Fig. 2. The peak attenuation varies about 1.8 $\mathrm{dB} / \mathrm{cm}$ and the minimum phase speed varies by about 100 $\mathrm{m} / \mathrm{s}$. Again, the ability to acoustically detect the effects of
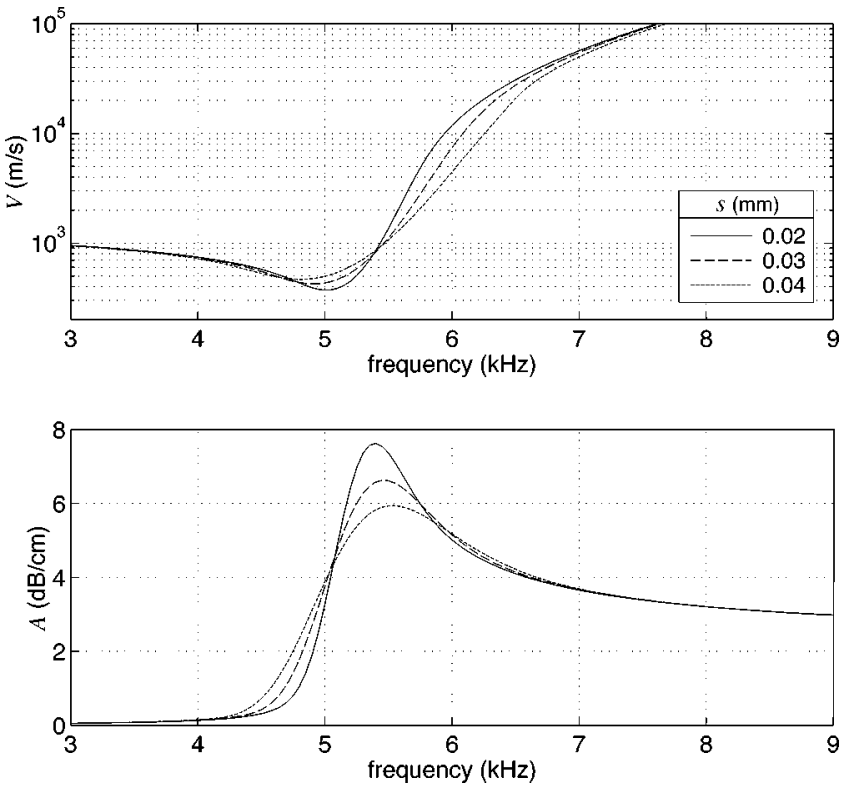

FIG. 2. The sensitivity to the distribution width is demonstrated. Phase speed (upper) and attenuation (lower) is shown for void fraction $\beta=10^{-4}$ and three standard deviations $s$. The legend applies to both plots. Additional bubble population details are given in the text.

these distribution changes may be greater than the ability to detect and control the bubble population parameters in an experiment.

\section{DESCRIPTION OF EXPERIMENT}

An overview of the measurement procedure is given first and then the various subsystems will be described in greater detail. The bubbles were generated directly at the measurement plane of the impedance tube, by either a single needle, which was lowered down into the impedance tube, or with a bubble injection manifold (BIM) that fit inside the impedance tube and deployed multiple needles for higher void fractions. The needle and the BIM can be moved between the impedance tube and two other systems that were used to measure the bubble size distribution and the overall void fraction. The experimental procedure was composed of four subprocedures. The first subprocedure was the calibration of the impedance tube. The needle or BIM was then installed in an optical apparatus for the measurement of the bubble size distribution. When the needle was used, the void fraction was also measured in the optical apparatus. When the BIM was used, it was moved to a third apparatus for void fraction measurement. Finally, the needle or BIM was installed in the impedance tube and the surface impedance of the bubble layer was measured.

A schematic of the impedance tube system is shown in Fig. 3. Its construction, calibration, and operation have been described previously. ${ }^{10,12}$ Briefly, the system consists of a heavy-walled stainless steel tube $(5.178 \mathrm{~cm}$ inner diameter, $2.541 \mathrm{~cm}$ wall thickness) with two custom fabricated wallmounted hydrophones. The system was designed to admit waves that are plane (to a high degree of approximation), despite some coupling between the fill material and the tube wall. Acoustic excitation was provided by a Kildare Corporation TP-400/A piston source at the lower end. The bubbly 


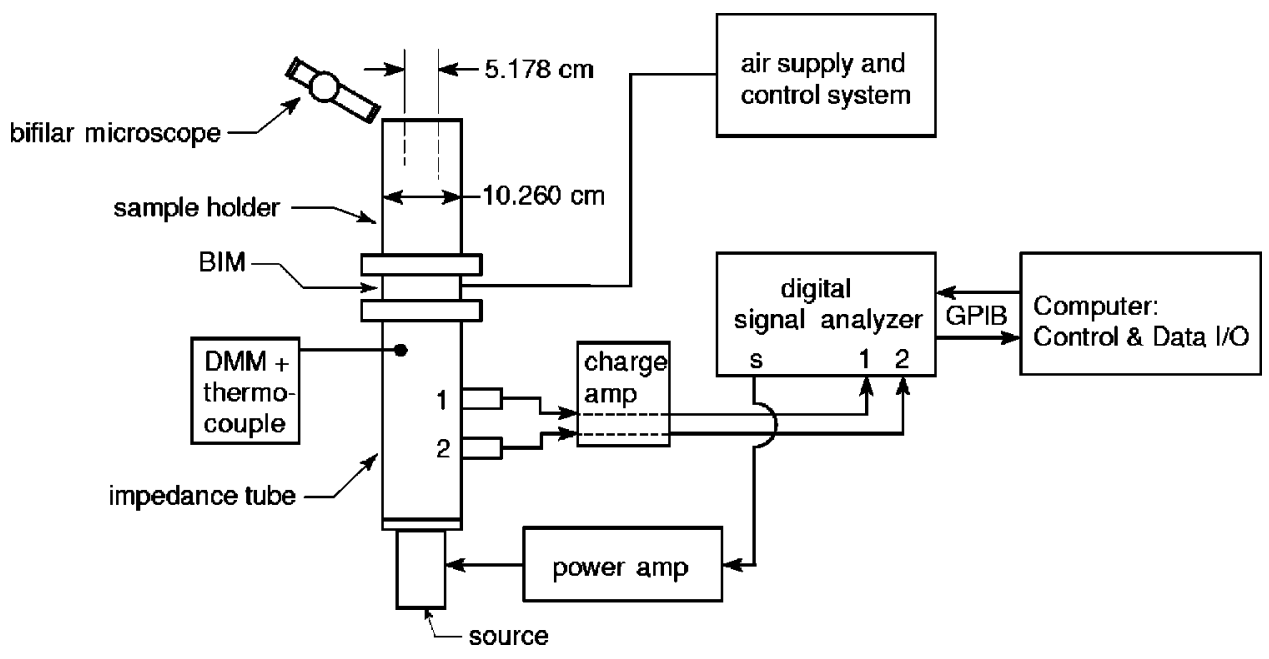

FIG. 3. The impedance tube system is shown in a schematic. The bifilar microscope is used during a calibration to measure the water height within the sample holder. The temperature of the impedance tube is monitored with a thermocouple and a digital multimeter. The remaining elements are discussed in the text.

liquid was contained within a sample holder mounted at the upper end. Signals were generated and received with a Hewlett-Packard HP89410A vector signal analyzer. The excitation signal was amplified by a Crown CE-1000 power amplifier and the hydrophone signals were conditioned with a Brüel \& Kjær model 2692 charge amplifier. The transfer function between the two hydrophones was measured with the HP89410A and the data was transferred to a laptop computer for storage and processing. The effective plane wave surface impedance of the bubbly liquid is related to the measured transfer function using Eq. (1) of Ref. 10 and a set of calibration functions obtained in the calibration procedure.

\section{A. Inversion procedure}

In the present work, the high attenuation provided by the bubbly liquid suppressed reflections from the air-water interface at the top of the sample holder, giving it an effectively infinite length. Therefore, plane progressive waves crossed into the bubbly liquid and the complex sound speed (phase speed and attenuation) was available directly from the measured surface impedance. This condition existed for void fractions as low as $O\left(10^{-5}\right)$, where on-resonance attenuation was $O(2) \mathrm{dB} / \mathrm{cm}$. The sample holder used in these experiments was $14 \mathrm{~cm}$ in length and, therefore, minimum round trip attenuation was about $56 \mathrm{~dB}$, which was sufficient to render the reflected signal indistinguishable from the background noise.

The phase speed and attenuation were obtained in the following way: Since $z_{s}=\rho c$ for the plane progressive waves incident on the surface of the bubbly liquid and $k=\omega / c$, the wave number $k$ in the bubbly liquid is given by

$$
k=\frac{\omega \rho}{z_{s}}
$$

where $z_{s}$ is the measured impedance and $\rho$ is obtained from the mixture density relationship $\rho=(1-\beta) \rho_{l}+\beta \rho_{g}$. Finally, the phase speed is given by

$$
V=\frac{\omega}{\operatorname{Re}[k]},
$$

and the attenuation coefficient is given by

$$
A=20 \log _{10}(e) \operatorname{Im}[k],
$$

in $\mathrm{dB}$ /unit length.

The key assumption used in this analysis is that only a single plane wave mode exists in the sample holder at a given frequency. For waves propagating within a liquid-filled waveguide with elastic walls, at least two modes can be present at all frequencies. Both modes can have longitudinal and radial components, but the lowest-order mode approaches a true plane wave mode as the wall impedance increases. ${ }^{13}$ This impedance tube was designed such that when filled with bubble-free water, both the radial component of the plane wave mode and the higher-order mode are negligible and can be ignored. ${ }^{10}$ Below resonance, the bubbly liquid medium is acoustically soft in comparison to pure water and the elastic waveguide effects are minimized even further. Above resonance, the bubbly liquid is acoustically hard in comparison to pure water and the elastic waveguide effects are more prominent. An inversion scheme that properly accounts for both components of both modes does not exist and for now these elastic waveguide effects have been ignored. This will be discussed further in Sec. IV C.

\section{B. Bubble production}

The bubby liquid consisted of air bubbles injected into distilled water using 30 gauge needles. The air was supplied from a compressed air bottle, through a primary regulator, then into a needle valve, a flow meter, and finally into the needle/s, either directly or through a manifold. A pressure gauge was connected between the flow meter and the needles. The flow rate and pressure measurements were used solely to ensure that the flow conditions, and hence the bubble population, remained as constant as possible throughout any given experiment. The bubble size distribution and overall void fraction were measured directly for each case.

For the first measurement presented in Sec. IV B, a single long needle (30 gauge, $0.31 \mathrm{~mm}$ diameter, $20.3 \mathrm{~cm}$ in length) was used. The needle was inserted into the sample holder from the open end of the tube and was positioned such that the bubbles were released at the measurement plane. Since changing the flow rate of air through a single needle changes the bubble size as well as the void fraction, one must add additional needles with the same per needle flow rate in order to independently increase the void fraction. 


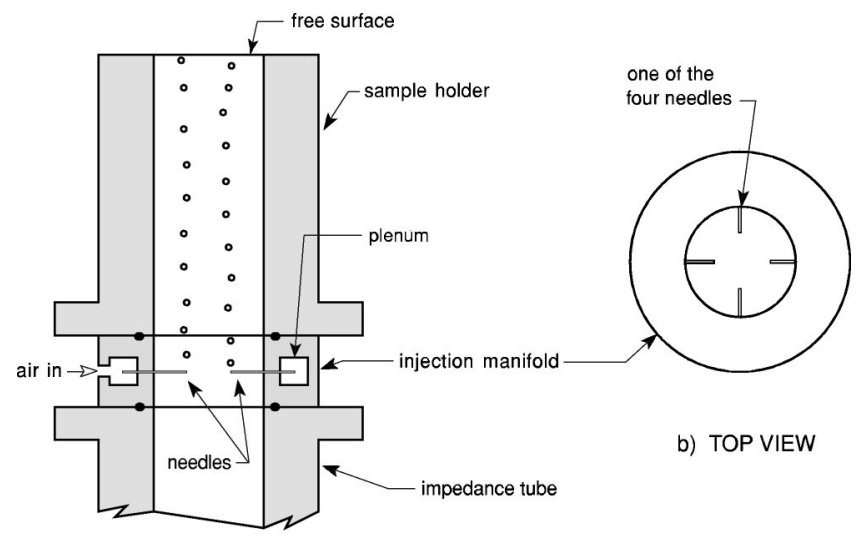

a) CROSS-SECTION

FIG. 4. A schematic diagram of the bubble injection manifold is shown. Part (a) is a cross-sectional view showing the injection manifold mounted between the impedance tube and the sample holder. Two needles are shown making bubbles. Part (b) shows a top view of the injection manifold separated from the impedance tube and the sample holder, with four needles in place. The needles were 30 gauge, $2.86 \mathrm{~cm}$ in length, and projected $1.27 \mathrm{~cm}$ into the tube.

Therefore, a bubble injection manifold (BIM) was developed to accurately position four 30 gauge needles at the measurement plane. The manifold body is of the same radial dimensions and material as the impedance tube itself, but contains an internal plenum that directs the air supply into the needles, as shown in Fig. 4(a). All the needles are coplanar with the impedance measurement plane. As the bubbles rose, they typically deviated from a directly upward path, yet they remained uniformly distributed along the longitudinal axis of the tube, and therefore the void fraction remained uniform in the direction of propagation. All but the first measurement presented in Sec. IV B were made using the BIM.

\section{Measurement of bubble size}

The bubble size distribution was determined by the direct photographic observation of the bubbles. Illumination was provided using a combination of direct and backlighting such that the outline of the bubbles appeared dark against a much lighter background. The single long needle or the BIM was placed within a small glass aquarium filled with distilled water. The height of water above the opening of the needles was the same as when installed in the impedance tube. The bubbles were free to rise up through the water column and they were photographed using a Kodak DC265 digital camera equipped with a macrolens and a strobe. The camera's maximum acquisition rate of about one photograph per $15 \mathrm{~s}$ was used. A machinist's scale located in the focal region was also photographed to provided a length reference. A schematic of the bubble size measurement system is shown in Fig. 5. Both the imaging and subsequent analysis were undertaken following guidelines published by The National Institute of Standards and Technology. ${ }^{14}$ A typical image obtained with this system is shown in Fig. 6(a) and the results of the image analysis are shown in Fig. 6(b). Note the relatively homogeneous distribution of bubbles both vertically and horizontally. A Cannon model GL1 digital video camera

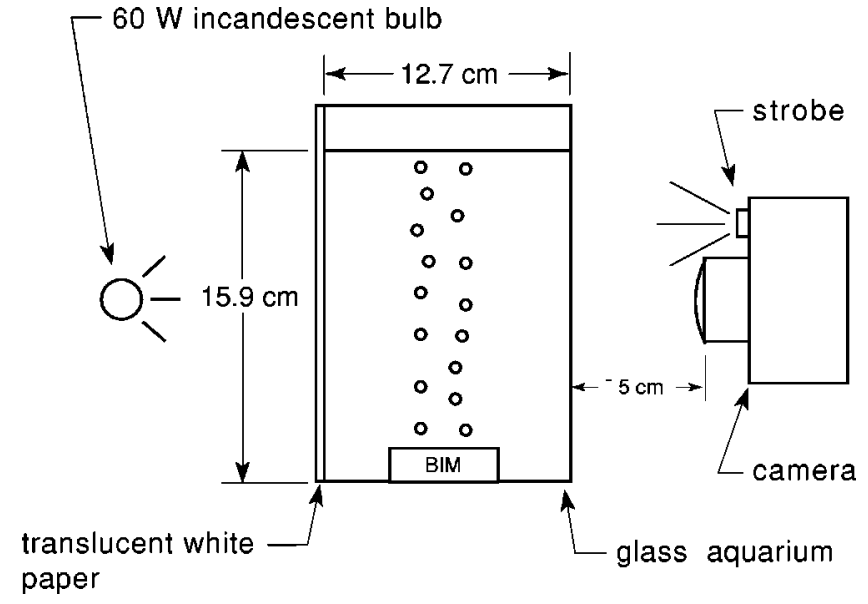

FIG. 5. A schematic diagram of the bubble size measurement system is shown with the BIM producing bubbles. The height of the water column is the same as that inside the sample holder during impedance measurements. Not shown is the machinist's scale used for a length reference.

was used to obtain photographic data at the higher rate required for the time-resolved measurements in Sec. V.

The bubbles exhibited a shape well approximated by an oblate spheroid. The images were processed with the image analysis software package NIH image. The major and minor axes $2 b$ and $2 c$ appeared in the plane of the image, and were determined by the NIH image in number of pixels. The length reference $l_{\text {ref }}$ (length/pixel) was taken from the machinist's scale. The spheroidal volume is given by $V$ $=\frac{4}{3} \pi b^{2} c l_{\text {ref. }}^{3}$. The effective spherical radius $a$ was then calculated based on an effective spherical volume,

$$
a=\left(\frac{3 V}{4 \pi}\right)^{1 / 3}=l_{\text {ref }}\left(b^{2} c\right)^{1 / 3} .
$$

The number of individual bubbles analyzed varied from experiment to experiment, but a typical number was 200 .

Two primary factors that contribute to the uncertainty of the bubble size measurement are a deviation of the actual bubble shape from the model shape, and the limited length

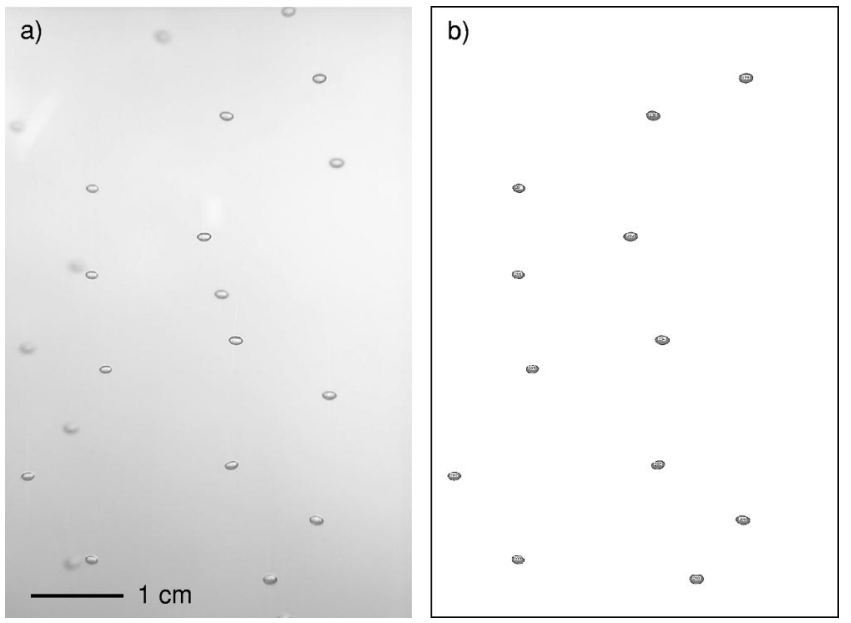

FIG. 6. A typical image obtained with the bubble size measurement system and four needles is shown in (a) along with a $1 \mathrm{~cm}$ scale bar. The same image after processing is shown in (b). The background was removed, each bubble was outlined, assigned a number, and the size data was stored in a file. 


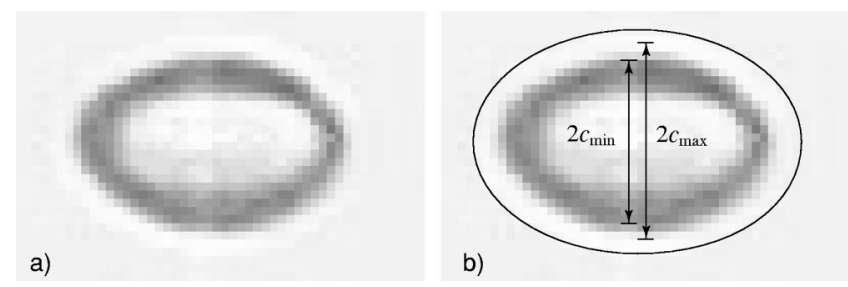

FIG. 7. An image of a typical bubble obtained by the imaging system is shown greatly enlarged in (a). Uncertainty due to pixelation is shown in (b). Equation (17) yields an effective spherical radius of $0.64 \pm 0.05 \mathrm{~mm}$ for this bubble.

resolution of the pixelated images. Close scrutiny of a magnified image of a typical bubble reveals that the error due to pixelation is likely the greater of the two. This is demonstrated in Fig. 7. An image of a typical bubble used in this work is shown greatly enlarged in Fig. 7(a), so that individual pixels can be seen. The same bubble is again shown in part Fig. 7(b), but a slightly larger ellipse has been overlain and it is apparent that the shape is a good match to that of the bubble. There is some uncertainty about the boundary of the bubble, though. When the image was digitized, the pixels were assigned discrete values of brightness; therefore one cannot be sure exactly where the boundary was. The motion of the bubble during the exposure also contributes to this uncertainty. Consider the minor axis of the bubble, with length $2 c$. If one assumes that the actual boundary lies somewhere between the outermost two pixels, then the minimum and maximum possible minor diameters are shown in the figure. For this bubble, $2 c_{\min }=20$ pixels and $2 c_{\max }$ $=24$ pixels. Although not shown in the figure, a similar situation exists for the major axis, with $2 b_{\min }=32$ and $2 b_{\max }$ $=36$ pixels, and for the length value, $1 / l_{\text {ref }}=230$ $\pm 2 \mathrm{pixel} / \mathrm{cm}$. Using Eq. (17) one finds the uncertainty in the measured effective spherical radius for this bubble is $a$ $\pm 7.7 \%$.

At this point a tradeoff is evident. For a given imaging system, one can increase the magnification and thereby increase the length resolution, but then the number of bubbles per image and the size of the statistical population is decreased. For fixed magnification, the uncertainty in bubble size is dependent on the bubble size. The uncertainty reported above was typical for this work. The maximum uncertainty in effective spherical bubble radius and did not exceed $a \pm 11 \%$.

\section{Determination of void fraction and low-frequency phase speed}

For the single needle case, the bubbles were few enough in number that all could be counted individually and the total volume of air within the sample holder could be determined from the bubble size measurements.

When using the BIM, the void fraction was determined using a cylindrical acoustic resonator of length $L$, filled with the bubbly liquid of interest, and operating below the cutoff frequency of the first higher-order mode of the tube and well below the resonance frequency of the largest bubble. In this range, known as Wood's limit, the low-frequency mixture

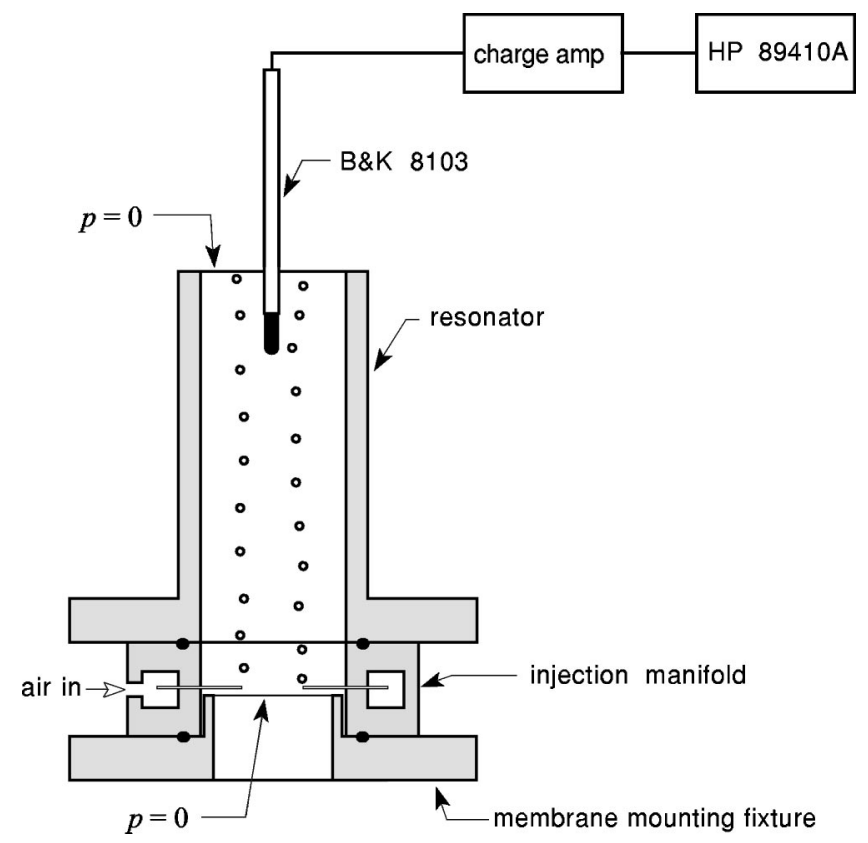

FIG. 8. A schematic diagram of the void fraction resonator is shown.

sound speed $c_{m l f}$ is dependent on void fraction $\beta$, as given by the relation ${ }^{15}$

$$
\frac{1}{c_{m l f}^{2}}=\frac{(1-\beta)^{2}}{c_{l}^{2}}+\frac{\beta^{2}}{c_{a}^{2}}+\beta(1-\beta) \frac{\rho_{a}^{2} c_{a}^{2}+\rho_{l}^{2} c_{l}^{2}}{\rho_{l} \rho_{a} c_{l}^{2} c_{a}^{2}},
$$

and $c_{a}$ and $\rho_{a}$ refer to the sound speed and density of air.

The resonator was modeled as a rigid-walled, openopen tube filled with a homogeneous liquid with sound speed $c_{m l f}$ and supporting only one-dimensional plane-wave modes. The fundamental resonance frequency is given by $f_{1}=c_{m l f} / 2 L$. The void fraction was determined from the measured frequency of the fundamental mode and Eq. (18). Sound speeds from the higher-order modes were also obtained. The modal sound speed was determined at each resonance frequency from $f_{n}=n c_{n} / 2 L$. Depending on the position of the hydrophone relative to the spatial acoustic field for each case, one, two, or three higher-order modes were observed.

A schematic diagram of the void fraction resonator is shown in Fig. 8. An aluminum tube $(30.13 \mathrm{~cm}$ length, 5.08 $\mathrm{cm}$ diameter, $0.635 \mathrm{~cm}$ wall thickness) was mounted to the top of the air injection manifold and sealed with an o ring. The lower pressure release boundary condition was achieved by closing the tube with a thin mylar membrane that was glued to a mounting fixture. The mounting fixture interfaces with the air injection manifold, sealing the tube with an o ring, and positions the membrane just below the needles. A Brüel and Kjær type 8103 hydrophone was typically positioned $2 \mathrm{~cm}$ from the open end of the tube. The hydrophone signal was conditioned with a Brüel and Kjær type 2692 low noise charge amp, which also provided bandpass filtering between $10 \mathrm{~Hz}$ and $10 \mathrm{kHz}$. The natural resonances of the tube were excited by the noise created by the bubbles pinching off the needles, in a manner similar to that reported by 
TABLE II. The table contains the results of the resonator-based void fraction measurements and the parameters used with Eq. (19) for calculation of the associated uncertainty. For Case 1, $\beta$ was obtained from optical measurements and its uncertainty is discussed in Sec. IV B.

\begin{tabular}{lcccc}
\hline \hline Case & 1 & 2 & 3 & 4 \\
\hline$\beta \times 10^{5}$ & 5.49 & 33 & 41 & 54 \\
$\Delta \beta(\%)$ & $\ldots$ & \pm 3.4 & \pm 3.2 & \pm 3.3 \\
$L(\mathrm{~cm})$ & $\ldots$ & 31.5 & 31.5 & 31.5 \\
$\epsilon_{L}(\mathrm{~cm})$ & $\ldots$ & \pm 0.2 & \pm 0.2 & \pm 0.2 \\
$f(\mathrm{~Hz})$ & $\ldots$ & 857.5 & 810.0 & 730.0 \\
$\epsilon_{f}(\mathrm{~Hz})$ & $\ldots$ & \pm 6.875 & \pm 6.125 & \pm 6.000 \\
$\epsilon_{c}(\mathrm{~m} / \mathrm{s})$ & $\cdots$ & \pm 7.8 & \pm 7.1 & \pm 6.7 \\
\hline \hline
\end{tabular}

Nicholas et al. ${ }^{16}$ The resulting hydrophone signal was acquired and acoustic spectra calculated with the HP89410A Vector Signal Analyzer.

Uncertainty $\epsilon_{L}$ in the length of the resonator and uncertainty in the resonance frequency $\epsilon_{f}$ due to the finite resolution bandwidth of the measured acoustic spectra lead to uncertainty $\epsilon_{c}$ in the measured sound speeds obtained from the resonator method and is given by

$$
\pm \epsilon_{c}= \pm 2\left(L \epsilon_{f}+f \epsilon_{L} \pm \epsilon_{L} \epsilon_{f}\right) .
$$

In turn, this leads to an uncertainty range $\beta \pm \Delta \beta$ in the void fraction, obtained by the satisfaction of Eq. (18) for $c_{m l f}$ $=2 L f_{1} \pm \epsilon_{c}$. Numerical values for these parameters are given in Table II.

\section{E. Bubble population statistics}

The bubble size data must be cast in the form of a probability density function $\wp(a)$ for input into the propagation model. Histograms of this data indicated that a truncated normal probability density function (PDF) was a suitable statistical model. Four parameters describe this distribution: the minimum radius $a_{\text {min }}$, the maximum radius $a_{\text {max }}$, the mean $a_{0}$, and the standard deviation $s$. The bubble radii data, obtained as described in Sec. III C, were ranked in ascending order. The minimum and maximum radii values were used as $a_{\min }$ and $a_{\max }$, respectively, and $a_{0}$ and $s$ were estimated by fitting a model cumulative distribution function (CDF), to an empirical CDF obtained from the measured radii.

The measured or empirical CDF is given by

$$
P_{\text {meas }}(a)=\frac{\bar{n}(a)}{N}, \quad a_{\text {min }} \leqslant a \leqslant a_{\text {max }},
$$

where $\bar{n}(a)$ is the number of observations with values less than or equal to $a$, and $N$ is the total number of observations. The model CDF is given by

$$
P(a)=\int_{a_{\min }}^{a} \wp(\xi) d \xi, \quad[0 \leqslant P \leqslant 1],
$$

where $\wp$ is PDF for the truncated normal distribution and is given by

$$
\wp(a)=\frac{1}{s \sqrt{2 \pi}} \exp \left[-\frac{\left(a-a_{0}\right)^{2}}{2 s^{2}}\right], \quad a_{\min } \leqslant a \leqslant a_{\max } .
$$
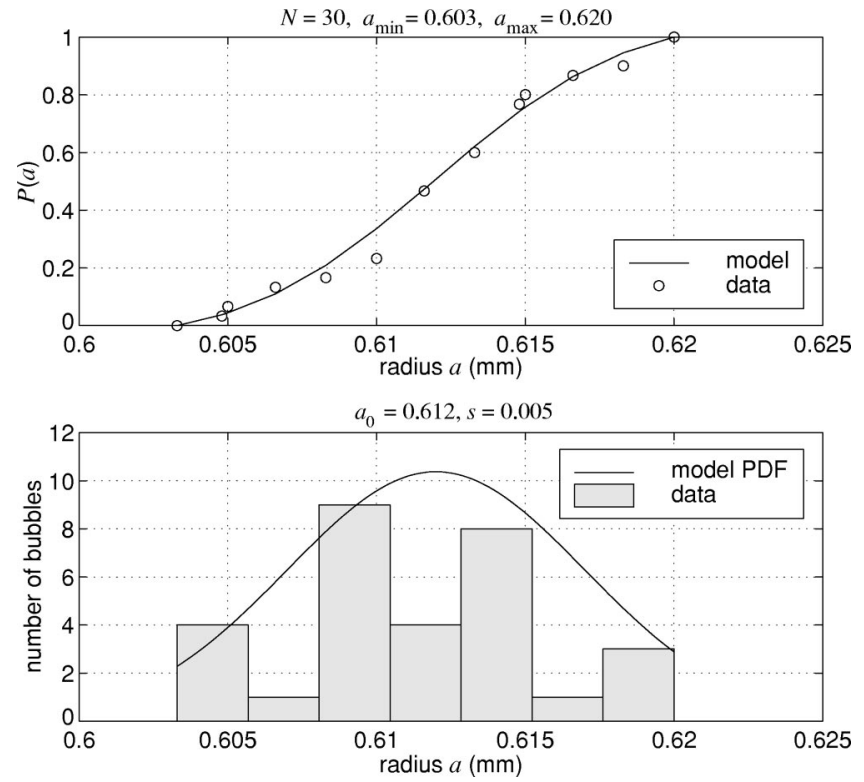

FIG. 9. The statistical data reduction is shown for bubbles created by a single needle. The upper frame shows the empirical CDF and the best-fit model CDF. The lower frame shows the resulting PDF and a histogram of the data, shown only for reference. The resulting distribution parameters are also shown (all radius units are in $\mathrm{mm}$ ).

Model CDFs were then computed for a range of $a_{0}$ and $s$ values, and a two-dimensional minimization of the chisquared parameter was performed, yielding the best-fit mean and standard deviation. The chi-squared parameter $\chi^{2}$ is given by ${ }^{17}$

$$
\chi^{2}=\sum_{i=1}^{N} \frac{\left(P_{\text {meas }}\left(a_{i}\right)-P\left(a_{i}\right)\right)^{2}}{P\left(a_{i}\right)},
$$

where $a_{i}$ is the $i$ th member of the rank ordered vector of size measurements. An example of this statistical analysis is shown in Fig. 9. The empirical CDF fitting method was used because it relied entirely on the data. The PDF can be fitted to a histogram of the data, but this requires an arbitrary choice to be made for the histogram bin size.

\section{F. Additional experimental details}

Two different types of experiments were conducted, time-averaged, and time-resolved. In both of these experiments, the goal was to measure the frequency-dependent phase speed and attenuation in bubbly liquid in the resonance regime. In the original experimental design, it was assumed that the bubble population statistics and the void fraction would be stationary in time, as long as the observable air injection parameters remained constant. Subsequent experimental observations revealed that the bubble population statistics were not stationary, even when observable bubble production parameters were stable. This type of behavior exhibited by needle manifolds has been reported before. ${ }^{18}$ Further, moving the bubble injection manifold from the bubble sizing apparatus, to the void fraction resonator, and finally to the impedance tube, resulted in slightly different bubble distributions, even when the observable flow parameters did not change. Because the bubble population parameters were changing, a relatively small degree of fitting was 
usually necessary in order to bring the time-averaged measurements and model into agreement, as discussed in Sec. IV. The discovery of nonstationarity lead to the idea that instantaneous, or time-resolved measurements should also be performed, as discussed in Sec. V.

\section{TIME-AVERAGED MEASUREMENTS}

\section{A. Procedure}

The procedure for the experiment was composed of four parts: impedance tube calibration, bubble size distribution measurement, void fraction measurement, and finally impedance measurement.

For both the calibration and the impedance measurements, transfer functions between the signals from the two impedance tube hydrophones were measured using the HP89410A vector signal analyzer. Pseudorandom noise was used as the excitation signal over a frequency range of 5-9 kHz. The hydrophone signals were Hann windowed and processed to yield a frequency resolution bandwidth of $2.5 \mathrm{~Hz}$. The calibration was performed first, using a procedure described in Ref. 10. Second, the single needle or the BIM was installed in the size distribution apparatus, shown in Fig. 5. The bubble size distribution was measured, as described in Sec. III C, which also yielded the void fraction for the single needle case. For the single needle case, bubble observation was limited to three photographs taken over a time frame of about $45 \mathrm{~s}$, yielding 30 bubble images. When the BIM was used, 16 photographs were taken over a time frame of about four minutes yielding about 200 bubble measurements. The BIM was then installed in the void fraction apparatus shown in Fig. 8, and the void fraction was measured, as described in Sec. III D. This step also produced some additional phase speed data below $5 \mathrm{kHz}$.

Finally, either the single needle was inserted into the impedance tube, or the BIM was installed [as shown in Fig. 4(a)], and the tube was filled with distilled water. The bubble production was then initiated and the airflow rate and pressure were allowed to equilibrate. These flow parameters were kept constant throughout the size distribution, void fraction, and impedance measurement procedures. Because noise is produced by the bubble generation, the acoustic drive level was dependent upon the experiment. The coherence function between the two wall-mounted sensors was monitored with the vector signal analyzer. The minimum power amplifier gain necessary to obtain a near-unity coherence function was used. This ensured a sufficient signal-to-noise ratio and a linear bubble response.

The impedance measurement was then conducted by obtaining 100 spectral averages of the transfer function between the two wall-mounted sensors, using the signal analyzer. The averaged transfer function $y$ was processed onboard the analyzer using

$$
y=\frac{G_{12}(f)}{G_{11}(f)},
$$

where $G_{12}$ is the averaged cross-spectrum given by
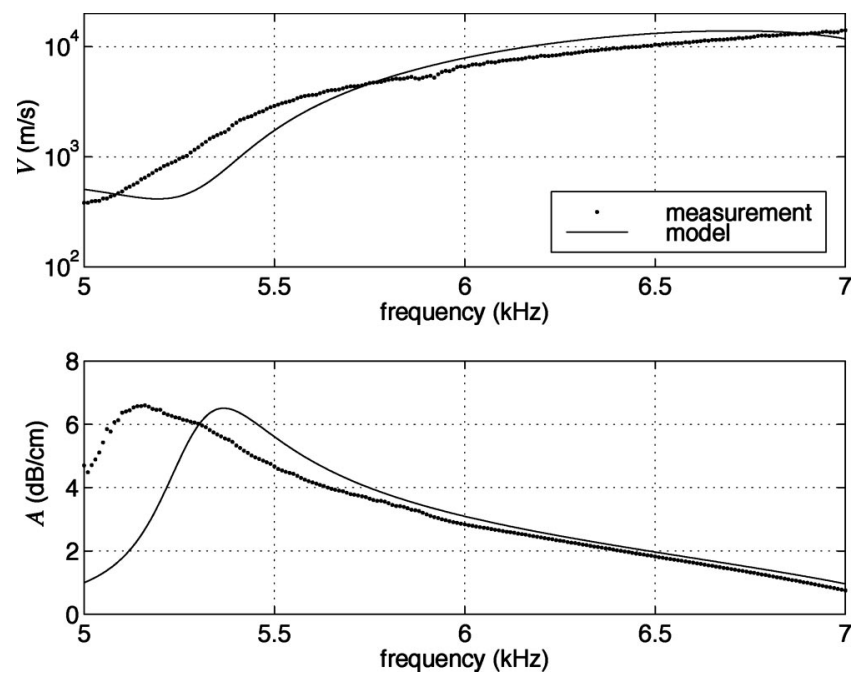

FIG. 10. Measured and predicted phase speed $V$ and attenuation $A$ for case 1, using the population parameters from Fig. 9.

$$
G_{12}(f)=\frac{1}{N} \sum_{n=1}^{N} S_{2, n} S_{1, n}^{*}
$$

$G_{11}$ is the averaged autospectrum, given by

$$
G_{11}(f)=\frac{1}{N} \sum_{n=1}^{N} S_{1, n} S_{1, n}^{*},
$$

and individual spectra $S_{i}$ are given by

$$
S_{i}(f)=\operatorname{FFT}\left[W(t) p_{i}(t)\right] .
$$

In the above expressions, FFT is the fast-Fourier transform, $W(t)$ is the Hann window function, $p_{i}(t)$ is the acoustic pressure signal at the $i$ th sensor position, the $*$ indicates the complex conjugate, and $N=100$ is the number of averages. Finally, the averaged transfer function $y$ was sent to a computer over GPIB and the impedance was calculated off-line, as described in Ref. 10.

The above procedure was repeated for a total of four different void fractions, using a single long needle and four needles installed in the BIM. Note that when the single long needle was used, the BIM was not installed. The sample holder and the impedance tube were bolted directly together.

\section{B. Results}

The results for the single needle (case 1) are presented first. The results of the bubble population analysis is shown in Fig. 9. In the top part of the figure, the measured and the model CDFs are shown, along with the population size $N$, and the minimum and maximum bubble radii. In the bottom part of the figure, the best-fit model PDF is shown along with its parameters $a_{0}$ and $s$. A histogram of the bubble size data is also shown for reference, although it was not used in the analysis. The measured void fraction was $\beta=5.49 \times 10^{-5}$.

The phase speed and attenuation measured for this bubbly liquid is shown in Fig. 10, along with the theoretical predictions given by Eq. (2), where $\wp(a)$ is given by Eq. (12) and the four bubble population parameters from Fig. 9. A complete list of the parameters used in the production of Fig. 10 is given in Table III. The measured data and the 
TABLE III. The table contains the parameters used to produce Figs. 10-15. All the physical parameters listed in Table I, except $c_{l}$, were also used. The bubble-free liquid sound speeds $c_{l}$ were measured inside the impedance tube and show variation due to temperature. The presence of the asterisk (*) in a cell indicates that the parameter was fitted.

\begin{tabular}{|c|c|c|c|c|c|}
\hline Case & 1 & 1 & 2 & 3 & 4 \\
\hline Figure & 10 & 11 & 13 & 14 & 15 \\
\hline$\beta\left(\times 10^{-5}\right)$ & 5.49 & $6.20 *$ & 33 & 41 & 54 \\
\hline$c_{l}(\mathrm{~m} / \mathrm{s})$ & 1455.5 & 1455.5 & 1457.4 & 1455.8 & 1458.0 \\
\hline$a_{\min }(\mathrm{mm})$ & 0.603 & $0.627^{*}$ & $0.580^{*}$ & $0.580^{*}$ & $0.580^{*}$ \\
\hline$a_{0}(\mathrm{~mm})$ & 0.612 & $0.636^{*}$ & $0.600^{*}$ & $0.622 *$ & $0.638^{*}$ \\
\hline$a_{\max }(\mathrm{mm})$ & 0.620 & $0.645^{*}$ & $0.750^{*}$ & $0.710 *$ & $0.750^{*}$ \\
\hline$s(\mathrm{~mm})$ & 0.005 & 0.005 & $0.031^{*}$ & $0.038^{*}$ & $0.035^{*}$ \\
\hline
\end{tabular}

model are qualitatively similar, but they are shifted by about $2 \mathrm{kHz}$ in frequency. Recall that the bubble size uncertainty is about $\pm 8 \%$, and that the size data and the acoustic data are not taken at the same time. Taking this into account by adjusting the radius so that the frequency of the measured and predicted peak attenuation are the same yields Fig. 11, where the radii $a_{\min }, a_{0}$, and $a_{\max }$ were multiplied by 1.04 . The volume-based void fraction estimate was also multiplied by $(1.04)^{3}$, yielding $\beta=6.2 \times 10^{-5}$. Agreement is now much better across the entire frequency range. Because of the low attenuation at this void fraction, a reduced frequency range is shown. Outside of this range, the nonreflecting condition at the top of the tube was surely violated, which causes the inversion process to fail. Note that the data presented in this chapter has an inherent uncertainty associated with the impedance measurement of $\pm 2.2 \%$. ${ }^{12}$ Also note that other, less quantifiable sources of error are discussed in Sec. IV C.

The measurements obtained with the BIM are shown next. Four 30-gauge needles, $2.86 \mathrm{~cm}$ in length, were installed in the BIM for cases 2, 3, and 4. Each represents a different void fraction, which was achieved by slightly varying the overall flow rate. The needles were spaced $90^{\circ}$ apart around the circumference of the BIM, and projected $1.27 \mathrm{~cm}$ into the tube. A single bubble size distribution measurement
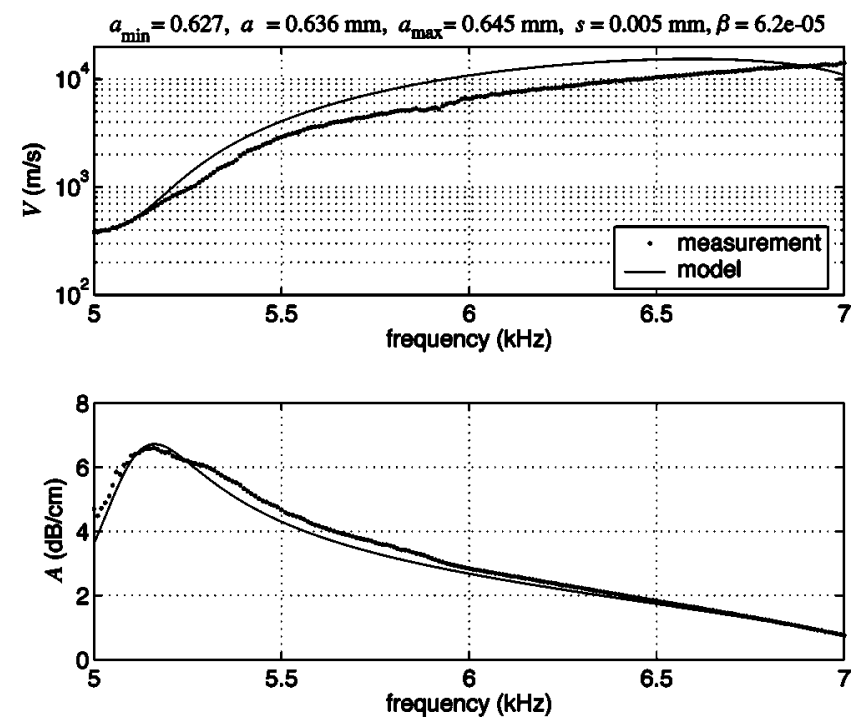

FIG. 11. The measured data for case 1 is shown again, but the predicted phase speed $V$ and attenuation $A$ was obtained by adjusting the bubble radius by $+4 \%$. The resulting bubble population parameters are also shown.
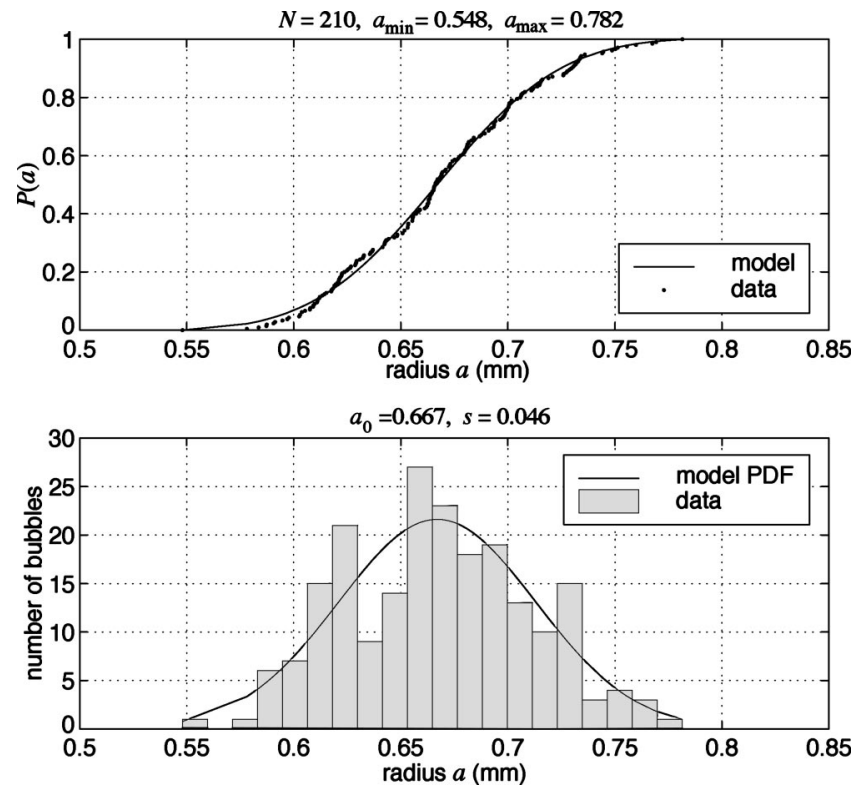

FIG. 12. The statistical data reduction is shown for bubbles created by the BIM with four needles and void fraction $\beta=41.0 \times 10^{-5}$. The upper frame shows the empirical CDF and the best-fit model CDF. The lower frame shows the resulting PDF and a histogram of the data, shown only for reference. The resulting distribution parameters are also shown (all units in $\mathrm{mm}$ ).

obtained with four needles and the intermediate flow rate is shown in Fig. 12. The measured phase speed and attenuation for cases 2, 3, and 4 are shown along with their associated predictions in Figs. 13, 14, and 15, respectively. A complete list of the model input parameters is given in Table III, and for convenience, the bubble population parameters are also shown in the figures.

In general, better agreement between the measurements and the model was found by fitting the bubble population statistical parameters within the uncertainty range of those measurements. Therefore, only the best-fit predictions are shown. Fitting the four bubble population statistics is easier
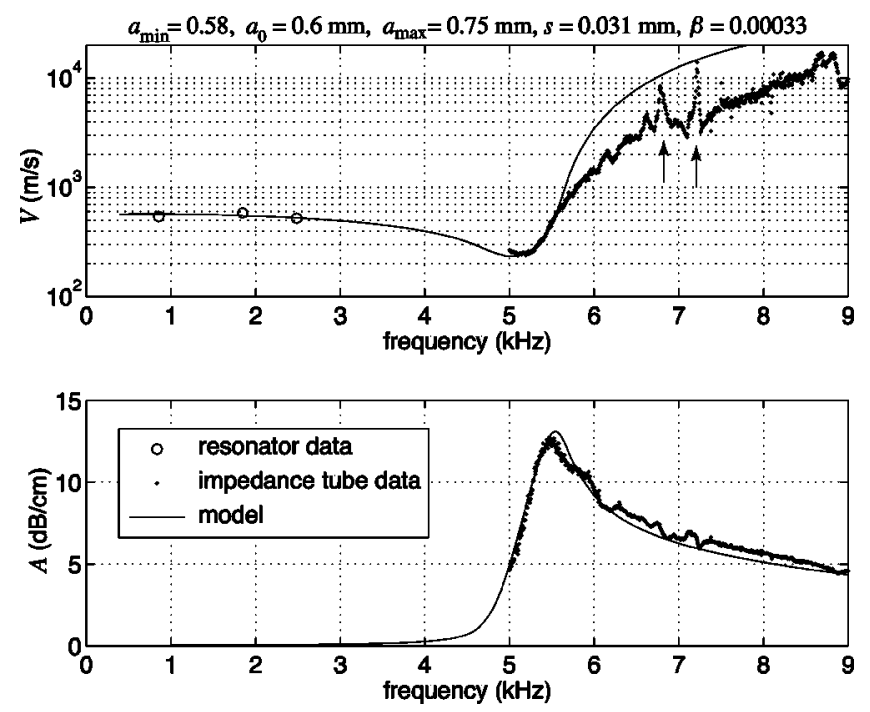

FIG. 13. The measured phase speed $V$ and attenuation $A$ for case 2 is shown. The predicted phase speed and attenuation that best fits the measured data is also shown along with the resulting bubble population parameters. The two vertical arrows near $7 \mathrm{kHz}$ indicate sharp fluctuations in phase speed that are discussed in the text. 

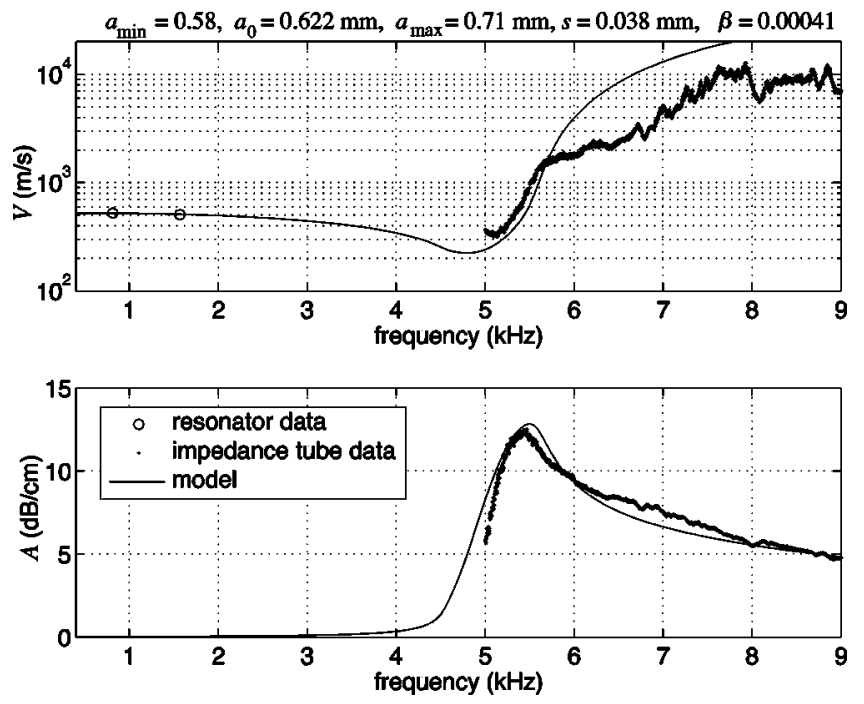

FIG. 14. The measured phase speed $V$ and attenuation $A$ for case 3 is shown. The predicted phase speed and attenuation that best fits the measured data is also shown along with the resulting bubble population parameters.

than one might expect because each parameter has a fairly unique effect. The fitting was done one parameter at a time, by hand. The difference between the data and the model in a certain range was minimized in a least squares sense by adjusting each parameter in turn. The mean bubble radius $a_{0}$ was fit using the frequency of peak attenuation. Standard deviation $s$ was fit using the slope of the attenuation curve on the low-frequency side of the peak attenuation. Minimum bubble radius $a_{\min }$ was fit using the width of the attenuation peak on the low-frequency side of the peak. Maximum bubble radius $a_{\max }$ was fit using the width of the attenuation peak on the high-frequency side of the peak. No attempt was made to automatically fit all four parameters simultaneously to the absolute minimum error across the frequency range. The region around resonance was given priority.
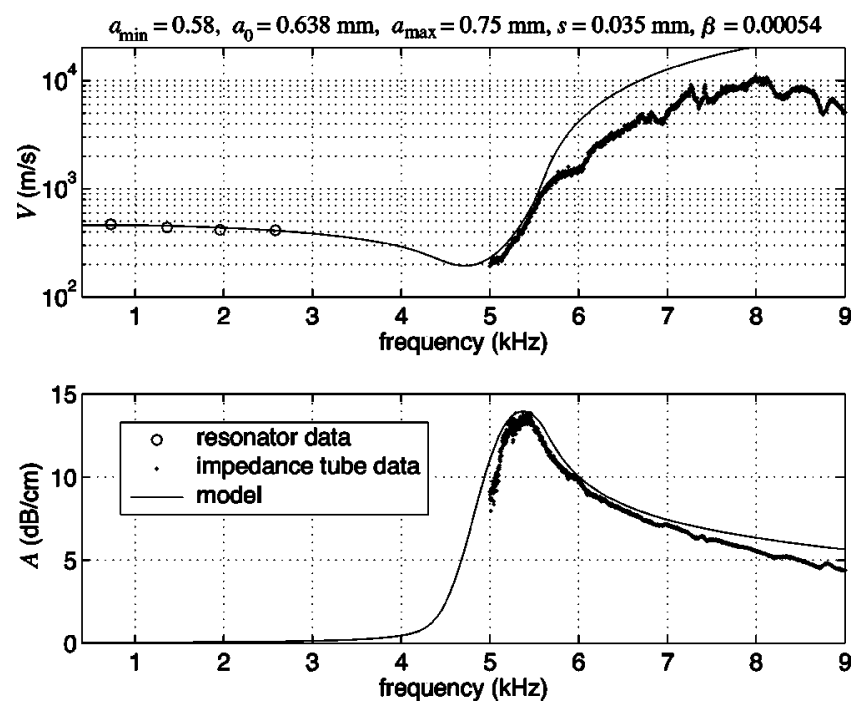

FIG. 15. The measured phase speed $V$ and attenuation $A$ for case 4 is shown. The predicted phase speed and attenuation that best fits the measured data is also shown along with the resulting bubble population parameters.

\section{Discussion of time-averaged results}

A number of general comments about the phase speed and attenuation data will be made and then these points will be discussed more fully. The predicted phase speeds above the resonance regime are generally higher than the measured values. The agreement is generally better for attenuation and is actually quite good. The deviation between the peak measured attenuation and the predicted value ranges from about 0.14 to $0.92 \mathrm{~dB} / \mathrm{cm}$. This agreement is better than that originally reported for peak attenuation in Ref. 3 , where the minimum data/model deviation was about $2.5 \mathrm{~dB} / \mathrm{cm}$ in Fig. 13 and was typically $10-15 \mathrm{~dB} / \mathrm{cm}$. The case $2-4$ measurements display sharp fluctuations that case 1 does not. The fitted bubble population was always narrower than the measured bubble population. The low-frequency phase speed measurements obtained from the VF resonator (the open circles in Figs. 13-15) agreed well with the resonance regime phase speed measurements obtained from the impedance; the theoretical predictions tied them together.

\section{Elastic waveguide effects}

Elastic waveguide effects were mentioned in reference to the impedance data inversion procedure in Sec. III A. It has been shown that the phase speed in a liquid-filled waveguide with elastic walls is reduced relative to the liquid's intrinsic sound speed and is dispersive. ${ }^{13}$ As the frequency increases through resonance, the bubbly liquid changes from acoustically soft to acoustically hard (relative to the bubblefree water) and the elastic waveguide effects should become more pronounced. This could explain the mismatch between measurement and prediction above resonance. The effect was investigated theoretically by starting with an idealized case. Propagation in the impedance tube was modeled using a elastic waveguide dispersion relation from the literature [Eq. (5) in Ref. 13]. In this lossless model, the modal phase speeds of waves propagating in the internal liquid are determined as a function of frequency, waveguide geometry, and the intrinsic (free-field) sound speed of the internal liquid. A frequency of $7 \mathrm{kHz}$ was used in the calculations. This frequency is above the bubble resonance frequency and a supersonic (relative to bubble-free water) phase speed is predicted for the unconfined bubbly liquid. This frequency is below the cutoff frequency for the first higher-order mode if this were a rigid-walled waveguide. For the elastic-walled case, two modes are predicted to propagate in the internal liquid and their phase speeds were calculated for a range of free-field sound speeds. Equation (5) of Ref. 13 is a lossless model, so the attenuation in the bubbly fluid was neglected. This is a major simplifying assumption, but the results are still useful for insight, if not absolutely accuracy.

These results are presented in Fig. 16. The predicted phase speed of each of the two modes is plotted as a function of the intrinsic bubbly liquid sound speed. In other words, according to the elastic waveguide model, if the internal liquid sound speed was that given on the $x$ axis; then the phase speed of each mode would be that given by the solid lines labeled "elastic mode 00 " and "elastic mode 01." The leading 0 indicates these are axisymmetric modes. If the waveguide was truly rigid, there would be only one mode and the 


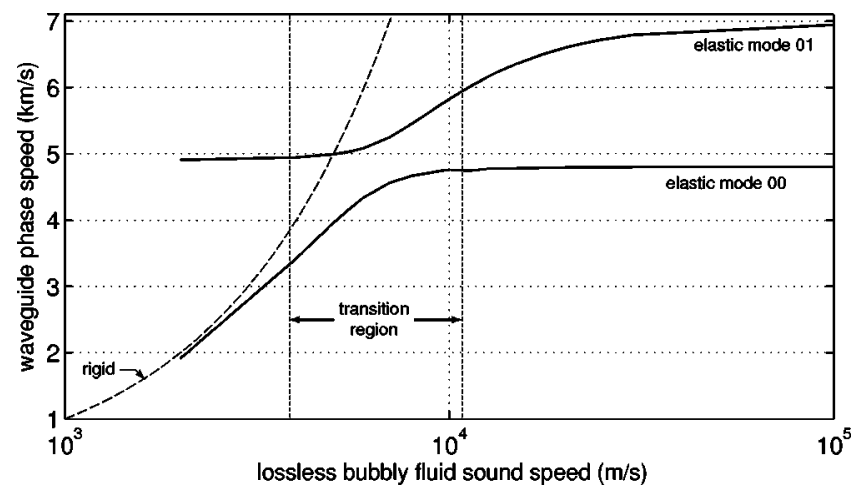

FIG. 16. The predicted phase speeds in an elastic waveguide are shown for supersonic regime bubbly fluid at $7 \mathrm{kHz}$. The mode labeled " 00 " is the plane wave mode, and " 01 " is the next higher-order mode. The dashed line represents the phase speed of the only mode present at this frequency for a rigid-walled waveguide.

waveguide phase speed would be the same as the intrinsic bubbly fluid sound speed. This case is represented by the dashed line labeled "rigid." (The plot would be straight on a linear scale but is curved because the $x$ axis is represented with a $\log$ scale.) The vertical dashed lines represent a transition region. Below this region, mode 00 has a phase speed close to the rigid waveguide sound speed and nearly plane wave fronts with little radial displacement. Mode 01 has curved wave fronts (concave down) and is not excited easily by plane disturbances. Above the transition region, both modes have curved wave fronts of almost the same concave up shape and similar radial components. Within the transition region, the phase speeds of the two modes are similar. Also notice that from midway through the transition region and higher in frequency, the two elastic mode speeds are always lower than the rigid-walled sound speed. This is a possible explanation why higher phase speeds are not measured, but it also brings up another issue.

According to the elastic waveguide model in Ref. 13, the 00 mode becomes nonplanar in the transition region. Now consider the situation within the impedance tube. An incoming plane wave begins to interact with the bubbly fluid at the interface between the tube and the sample holder and finds no suitable plane wave mode to excite. Part of the incident energy is reflected and the transmitted energy must get divided up into two propagating modes, and possibly a great number of evanescent modes. In such a case, the inversion that is used to extract phase speed and attenuation from the impedance measurements would break down, because it assumes only a single plane progressive mode for the transmitted wave.

Further evidence in support of this is available directly from the measured results. Consider the data in Fig. 11, which show a measured phase speed greater than $10000 \mathrm{~m} / \mathrm{s}$ at $7 \mathrm{kHz}$. According to Fig. 16, the maximum phase speed for mode 00 is less than $5000 \mathrm{~m} / \mathrm{s}$ and about $7000 \mathrm{~m} / \mathrm{s}$ for mode 01 . This is still below cutoff for the next higher-order mode, so nothing should be able to propagate at $10000 \mathrm{~m} / \mathrm{s}$. The existence of other modes must be interfering in such a way that an effective single mode phase speed is obtained from the inversion. An initial attempt was made using an inversion technique that accounts for the higher-order mode, but it was not successful. ${ }^{12}$ This remains an unresolved issue for phase speed measurements made with the present technique above resonance frequency, but plays a negligible role at resonance.

\section{Signal and noise issues}

The phase speed measurements exhibited an increase in sharp, rapid fluctuations with frequency when the BIM was used. The largest two of these features are indicated in Fig. 13 with small vertical arrows. The resulting increase in void fraction was accompanied by an increase in noise level produced as the bubbles broke off the needles. This noise represents energy entering the impedance tube from a direction not accounted for and, therefore, it could have corrupted the impedance measurements.

The measured coherence function was used to investigate this potential signal-to-noise issue. A coherence function greater than zero but less than unity implies one or a combination of the following: ${ }^{19}$ (1) The presence of extraneous noise in the measurements. (2) The output signal includes the result of excitation not measured by the input. (3) Some nonlinearity between sensors 1 and 2. (4) Resolution bias errors are present.

Items (3) and (4) were ruled out because coherence functions obtained from measurements with a bubble-free transmission line termination were always close to unity, except in narrow bands. These bands are associated with nulls in the field that happen to be co-located with one of the pressure sensors. The acoustic drive level was always well below the threshold for nonlinear effects in distilled water; there were no bubbles between the measurement sensors. The resolution bandwidth of the measurement was not changed when bubbles were introduced.

Items (1) and (2) were possibilities, though, and an experiment was done to investigate this. Impedance measurements were conducted for five signal-to-noise ratios (SNR), in increments of $10 \mathrm{~dB}$, with bubble production, and hence bubble-induced noise production constant. The SNR had no influence on the fluctuations. They did not increase as the SNR was decreased. At the lowest SNR, the coherence function was below 0.4 for much of the frequency range, but the rapid fluctuations did not deviate from the higher SNR cases. Therefore, excess bubble noise was ruled out as the cause.

What else could cause these perturbations? Perhaps it was the needles, or more specifically, the needles and the air inside. Case (1) shows none of these fluctuations. It was made with a single long needle lowered from the top. For the remaining cases, four needles were positioned parallel to and residing directly in the measurement plane. They present a larger cross section than did the long needle inserted from the top, and are positioned completely in the plane of interest. These are accompanied by an increased fluctuation level. This trend was observed to increase as the number of needles increased. ${ }^{12}$ The presence of needles in the measurement plane certainly deviates from a homogenous bubbly liquid and could possibly cause the fluctuations.

These fluctuations could also be caused by smaller bubbles attached to the ends of the needles. Before a bubble 

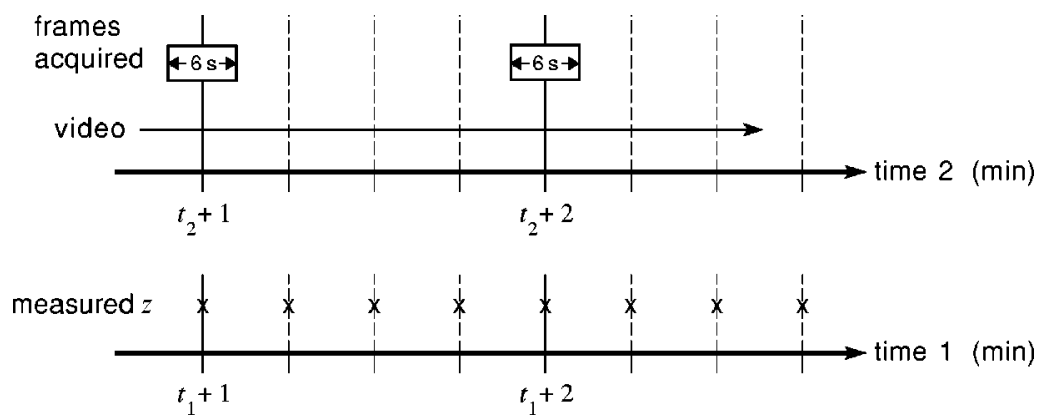

FIG. 17. Time lines for the time-resolved impedance measurements are shown. The impedance was measured in $66 \mu$ s segments once every $15 \mathrm{~s}$. These measurement times are shown by the symbol " $\times$." The video recorded continuously, as indicated with the thin horizontal arrow. Individual frames were acquired from the video for six second blocks every minute.

detaches from its needle and rises upward, it spends some time attached to the end of the needle during the growth phase. During that time, it is certainly being interrogated by the incoming sound wave and therefore contributing to the impedance of the bubbly fluid. Because the bubble is interrogated multiple times, the net effect is to increase the influence that particular bubble size has on the final results. A multiple delta function bubble distribution present at the measurement plane could possibly account for the sharp phase speed peaks and fluctuations.

\section{Bubble population parameters and void fraction}

When the BIM was used (cases 2-4), the bubble radii standard deviation required to fit the propagation model to the impedance tube data was smaller than the standard deviation obtained from the photographic data. It was subsequently determined that the bubble population statistics varied on a time scale of about two minutes, even when the observable bubble production parameters were stationary. Therefore, bubble size distributions (BSDs) observed optically over several minutes were wider than the best-fit model BSDs used to match acoustic data gathered over about $45 \mathrm{~s}$. Further, knowledge of the bubble population needs to be at least an order of magnitude more accurate to absolutely validate any propagation model. The bubble population parameter measurement should be conducted in situ with the impedance measurements. The low-frequency void fraction measurement technique proved to be reliable and agreement was found with both the resonance regime measurements and calculations. The in situ incorporation of the void fraction measurement would be ideal, but this task could be continued off-line. Finally, the increased control of bubble production would be beneficial, but if in situ population measurements are actually available, this is less important.

\section{TIME-RESOLVED EXPERIMENT}

An assumption made early in this work was that the experimental bubble populations would exhibit statistics that were stationary in time. As previously discussed, this was not the case. Since the bubble population characterization and the impedance measurement could not be done at the same time, an absolute comparison between the propagation model and experimental measurement was not possible. An investigation of the relative variability was possible and became the goal of the time-resolved experiment. In order to do this, the bubble population parameters and the acoustic impedance were sampled on similar time scales.

\section{A. Procedure}

The procedure for instantaneous impedance measurements was almost the same as that for the time-averaged measurements just described. Four subprocedures were again performed, but in the following order: calibration, void fraction measurement, impedance measurement, and then bubble size distribution measurement. The void fraction measurement procedures were unchanged from the time-averaged experiment.

The goal of this experiment was to observe the time variation of both the bubble size distribution and the propagation parameters. A periodic chirp was used as the excita-
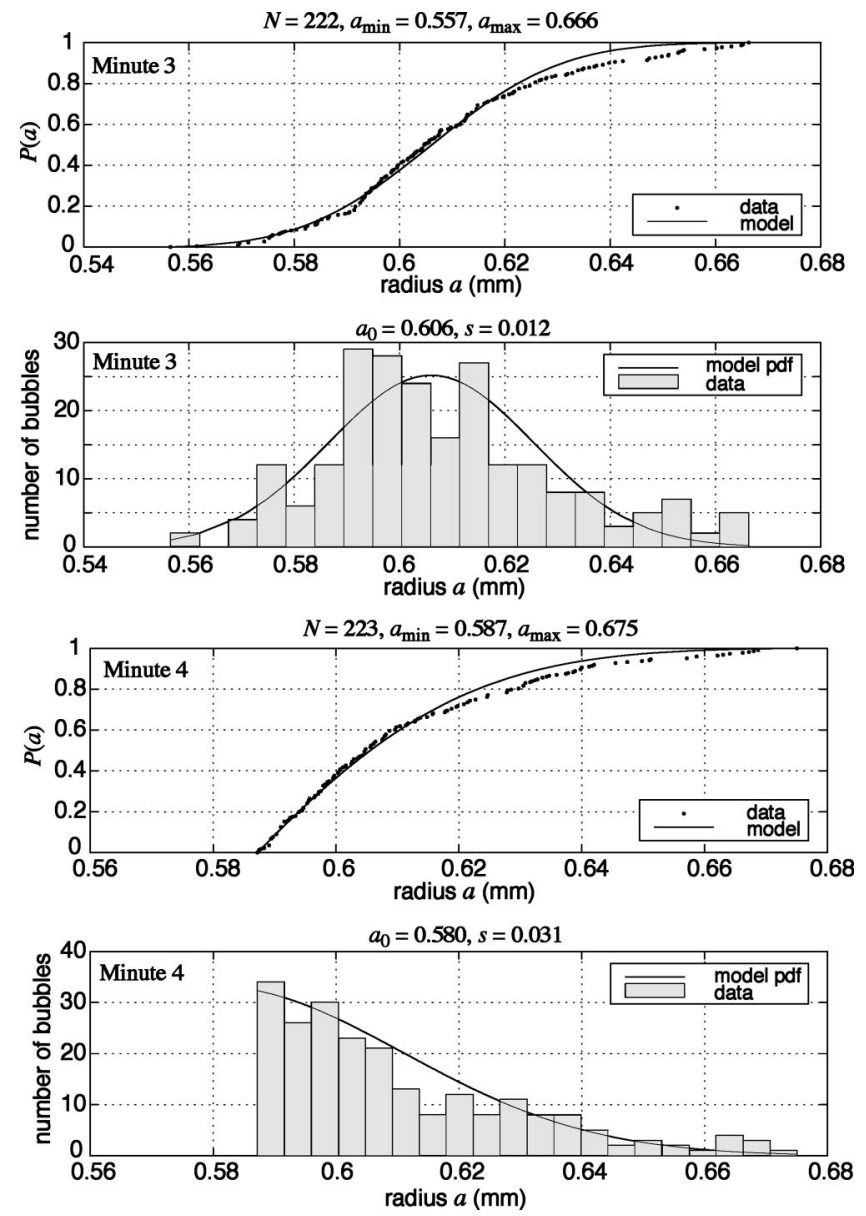

FIG. 18. Bubble population statistics at minutes 3 and 4 of the time-resolved experiment. For each case, the upper frame shows the empirical CDF and the best-fit model CDF. The lower frame shows the resulting PDF and a histogram of the data, shown only for reference. The resulting distribution parameters are also shown (all units in $\mathrm{mm}$ ). 

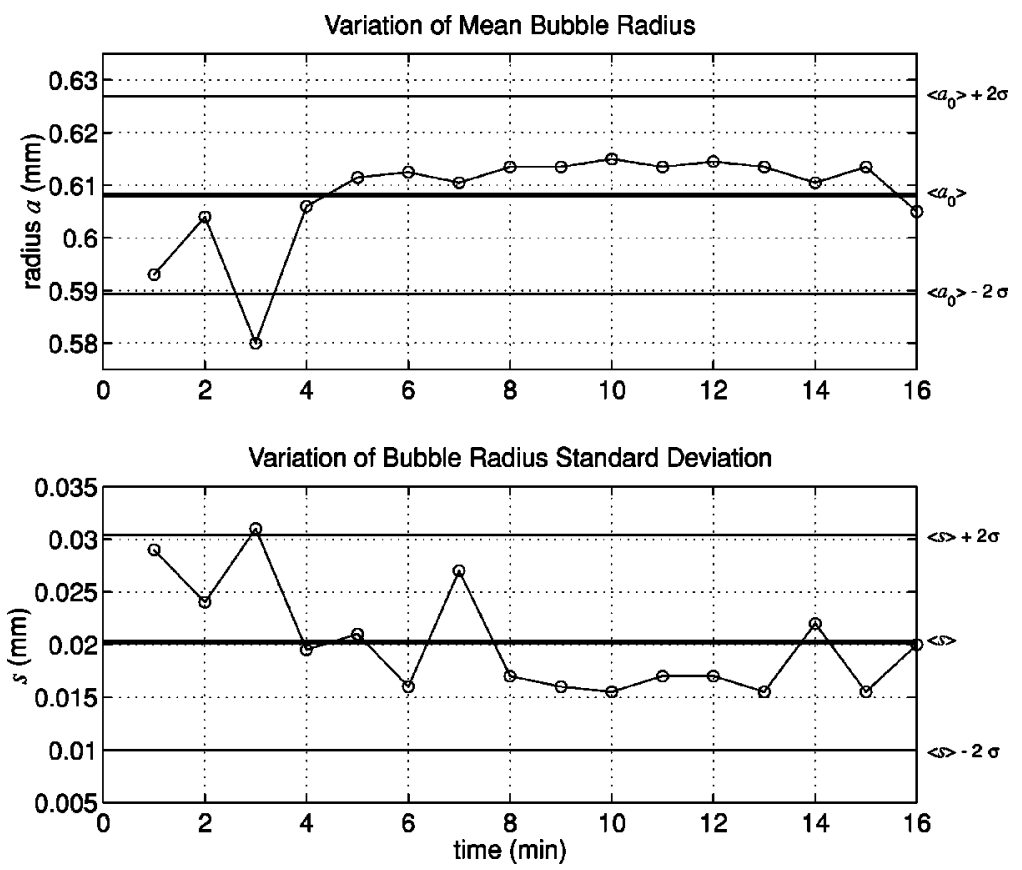

FIG. 19. Mean bubble radius $a_{0}$ and standard deviation $s$ are shown as a function of time. The thick horizontal lines represent the global means, $\left\langle a_{0}\right\rangle$ and $\langle s\rangle$, and the thin horizontal lines represent two standard deviations above and below the global means.

tion signal because it requires no averaging and could be used to obtain near-instantaneous measurements. The signal was periodic with respect to the data acquisition time window and was $66 \mathrm{~ms}$ in length. A Hann window was used, which resulted in a resolution bandwidth of $15 \mathrm{~Hz}$ for a frequency range of $4-10 \mathrm{kHz}$. The data from $4-5 \mathrm{kHz}$ and 9-10 kHz was discarded.

The calibration was performed using the periodic chirp, then the void fraction measurements were performed. The impedance measurement procedure began as it did in the previous section, but instead of taking 100 back-to-back averages, one chirp-excited impedance measurement was taken every $15 \mathrm{~s}$ for $16 \mathrm{~min}$. This was done for a single void fraction case, using the BIM and four needles.

The still camera could not operate fast enough to sample the bubble size distribution on the same time scale as the impedance, so a digital video camera was used. The resolution of this video camera was not high enough to image many bubbles in the same frame, so high magnification was used and one or two bubbles from a single needle were imaged instead. After the bubble production was initiated and the flow parameters were stabilized, video was recorded continuously for $16 \mathrm{~min}$, but only $6 \mathrm{~s}$ blocks taken every $60 \mathrm{~s}$ were processed. Therefore, $6 \mathrm{~s}$ of video for each event translated into 180 frames and about 220 bubbles per event. Under such circumstances, the bubble population is sampled photographically for bubble size in $331 / 3 \mathrm{~ms}$ intervals, and the impedance measurements sample $66 \mathrm{~ms}$ of the bubble population. A time line relating the impedance measurements and the size measurements is shown in Fig. 17. These images were all processed for bubble size, as described in Sec. III C.

\section{B. Results}

Using the technique given in Sec. III E, the bubble population statistics were obtained for each of the 16 populations. The CDF/PDF plots for the entire dataset are presented in Ref. 12 along with additional details about the data acquisi- tion and analysis. The uncertainty in these bubble size measurements was $\pm 11 \%$. The histograms of two neighboring bubble populations, generated one minute apart, are presented in Fig. 18. It is clear that the bubble population is changing dramatically on this time scale. The statistical bubble population parameters are plotted as a function of time in Figs. 19 and 20. The following notation and definitions were used. The global mean of a variable $x$ is given by $\langle x\rangle$, where

$$
\langle x\rangle=\frac{1}{16} \sum_{i=1}^{16} x\left(t_{i}\right),
$$

and the standard deviation of $x$ is given by

$$
\sigma=\left\{\frac{1}{16-1} \sum_{i=1}^{16}\left[x\left(t_{i}\right)-\langle x\rangle\right]^{2}\right\}^{1 / 2} .
$$

The variation of the mean bubble radius $a_{0}(t)$ and the radius standard deviation $s(t)$ is shown in Fig. 19. It may appear that a start-up transient was captured at the beginning of the upper plot, but recall that the observable control parameters of the bubble production process had reached steady state prior to the image acquisition and were not fluctuating. The curves shown in Figs. 19 and 20 confirm that the bubble population parameters are fluctuating dramatically and rapidly. Variation greater than two standard deviations can occur within a minute, and there is also evidence of long term trends, specifically in the standard deviation.

The void fraction, determined just prior to the impedance measurements using the resonator technique, was 3.9 $\times 10^{-4}$. Phase speed $V$ and attenuation $A$ were obtained from each impedance measurement. For compatibility with the bubble size data, $V$ and $A$ for four consecutive measurements were averaged together to represent one minute of time, then the next four were averaged, and so on, until 16 min of measured $V$ and $A$ were obtained. Predicted phase speeds and attenuations were then calculated using Eqs. (2) 


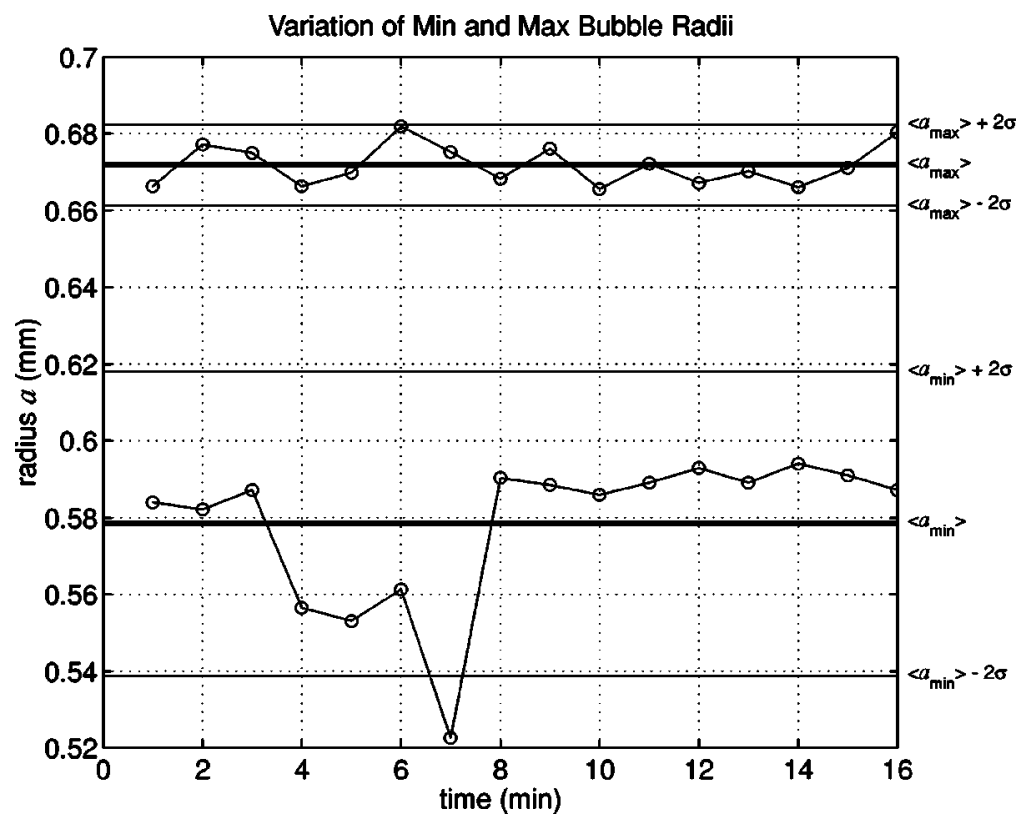

FIG. 20. Minimum and maximum bubble radius is shown as a function of time.

and (12), the measured bubble population parameters from Figs. 19 and 20, and the measured void fraction.

In order to compare the variability of these multifaceted measurements and calculations, attention will be focused on two parameters: the maximum attenuation $A_{\max }(t)$ and the frequency at which that maximum attenuation occurs, $f_{m}(t)$. Since the relative variation is of interest, the results will be presented in normalized form, $A_{\max } /\left\langle A_{\max }\right\rangle$ and $f_{m} /\left\langle f_{m}\right\rangle$, where the brackets represent the global mean as defined in Eq. (28), and the normalized standard deviation of parameter $x$ is given by

$$
\bar{\sigma}=\left\{\frac{1}{16-1} \sum_{i=1}^{16}\left[\frac{x\left(t_{i}\right)}{\langle x\rangle}-1\right]^{2}\right\}^{1 / 2} .
$$

The results are given in Figs. 21 and 22 for attenuation and frequency, respectively. When cast in this form, the predicted variation of the acoustic properties due to changes in the bubble population is quite similar to the observed variation. For the normalized frequency parameter, a $\bar{\sigma}$ variation of $0.87 \%$ is predicted and $0.84 \%$ is observed. For the normalized attenuation parameter, a $\bar{\sigma}$ variation of $4.9 \%$ is predicted and $5.3 \%$ is observed.

\section{Discussion}

The similarity between predicted and observed statistical variability of the peak attenuation and its frequency is quite good. Making comparisons of the relative, as opposed to absolute quantities minimized the problems relating to the
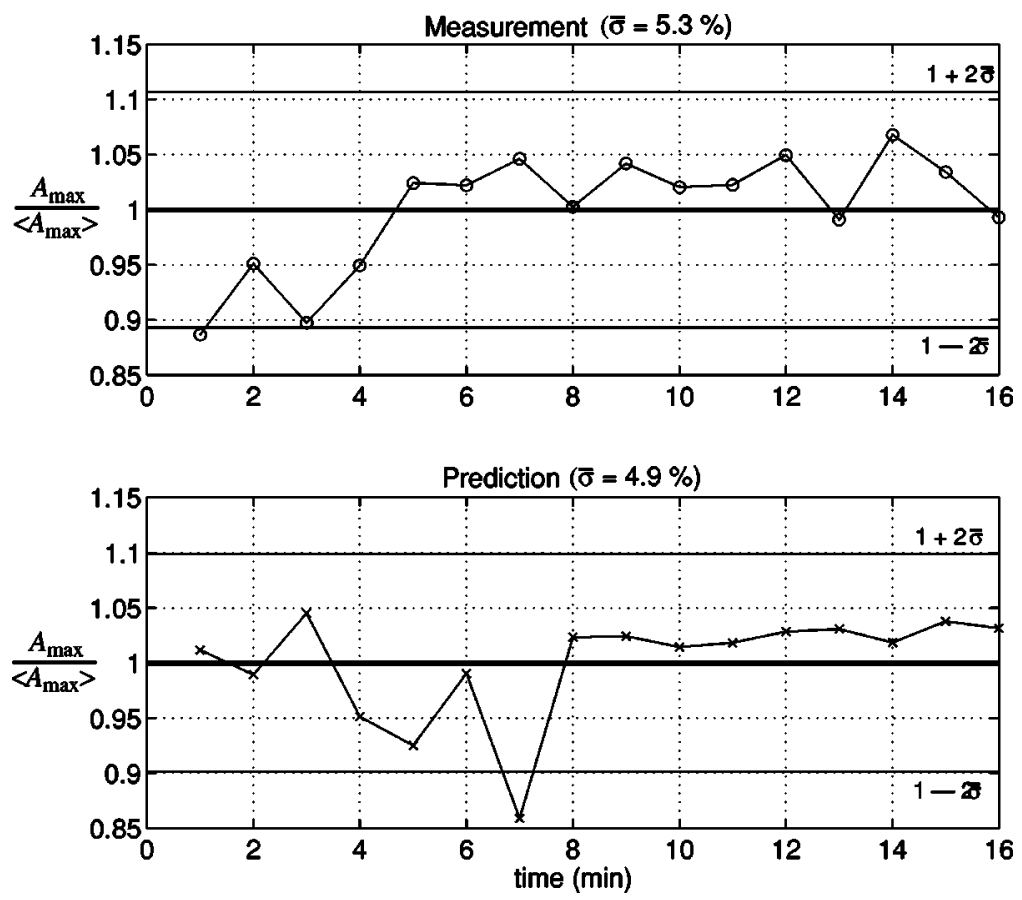

FIG. 21. Measured and calculated maximum attenuation is shown as a function of time, normalized by the global mean. 

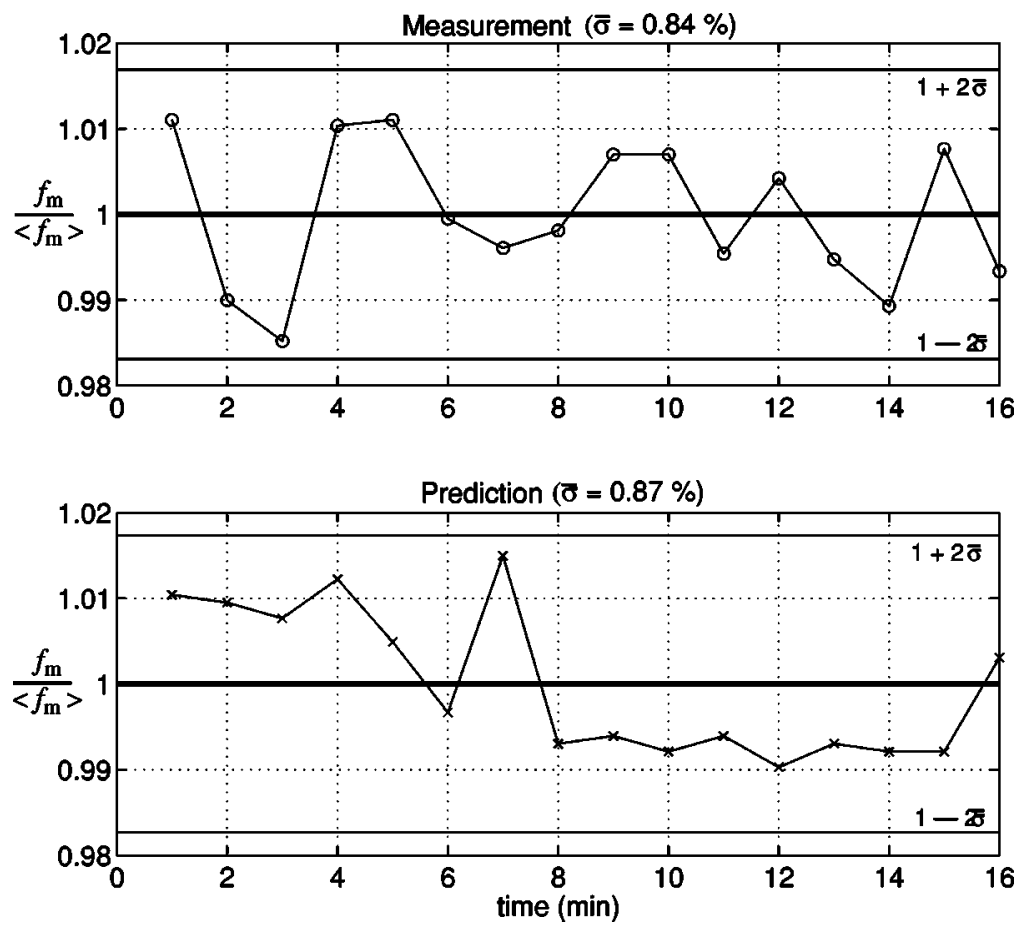

FIG. 22. Measured and calculated frequency of maximum attenuation is shown as a function of time, normalized by the global mean. uncertainty of the instantaneous bubble population, including the uncertainty in the absolute size of the bubbles.

In the region around peak attenuation, the phase speed is near its minimum, hence the elastic waveguide effects are minimized. Therefore, the inversion problems that are evident well above resonance play no role here. In addition to the inherent errors already discussed, there is an additional source of bias error for this experiment. The resolution bias error was made negligible in the time-averaged experiment, as confirmed by near unity measured coherences. The short excitation time in the present experiment lead to a resolution bandwidth of $15 \mathrm{~Hz}$, which increased the maximum estimated resolution bias errors a small amount. The level of error is still significantly smaller than the observed fluctuation, which can confidently be attributed to changes in the medium itself.

It has long been predicted from theory that propagation in bubbly fluids is sensitive to the bubble population parameters. The present work has reinforced this notion both theoretically and experimentally. The significance of these results is the following: Existing theory, specifically that represented by Eq. (2), is capable of predicting the relative variability of sound propagation parameters in bubbly liquids at resonance, if the bubble population parameters are known, up to a void fraction of $5.4 \times 10^{-4}$. This appears to be the case, despite the fact that the absolute accuracy of the model represented by Eq. (2) has not been verified experimentally.

\section{SUMMARY AND CONCLUSIONS}

In this work, the acoustics of bubbly liquid was investigated. Experiments were conducted that focused on linear plane wave propagation parameters in the frequency range surrounding individual bubble resonance. An impedance tube system was used to measure the phase speed and attenuation in fluids composed of air bubbles and distilled water for a range of void fractions between $6.2 \times 10^{-5}$ and $5.4 \times 10^{-4}$. The bubble size distributions were centered around $0.62 \mathrm{~nm}$ in radius. The void fraction and size distribution parameters were obtained from secondary measurements done just prior to or just after the measurement of impedance. It was found that the bubble population statistics were not stationary in time. They were changing during the experiments, even though the observable bubble production controls were stationary in time. Despite this discrepancy, it was found that the model discussed in Sec. II could be used to describe measurements near resonance. In order to do so, some of the bubble population parameters were used to fit the model to the measurements. This fitting was within the uncertainty of the population parameter measurements and yielded good agreement between the model and the observations near resonance for both phase speed and attenuation. This agreement is better than the agreement obtained between the model and measurements previously reported. ${ }^{3}$ Above resonance, the model overpredicted the observed phase speed, however, it was determined that the inversion technique used to obtain the phase speed and attenuation from the impedance measurements was not appropriate for use when the phase speed rose above approximately $2000 \mathrm{~m} / \mathrm{s}$. Therefore, further use of the impedance tube technique for an investigation of the supersonic regime above resonance will required additional work on the inversion process.

In a second round of experiments, the variability of both the bubble population statistics and the propagation parameters were investigated. The population statistics measurements and impedance measurements were still done in succession, but, here, the time scales of the two sets of measurements were much more similar than in the timeaveraged experiment. It was found that Eq. (2) could indeed predict the relative variability of the propagation parameters at resonance frequency, specifically the peak attenuation and 
the frequency at which it occurred. This is the case despite the fact that the absolute accuracy of Eq. (2) has not been experimentally verified. Another general conclusion drawn from these results is the following: the observation of acoustical parameters in bubbly liquids must be done on the same time scale as the observation of the associated bubble population parameters.

Finally, these results indicate that the absolute accuracy of Eq. (2) and of other competing theories can be verified near resonance using the existing impedance tube system. In order to do this, a colocated instrument to simultaneously measure bubble population statistics, or more stable bubble production equipment is required. In either case, the resolution and accuracy of the sizing apparatus must be increased by an order of magnitude over the present system, and the number of bubbles observed per unit time should also be increased by an order of magnitude.

\section{ACKNOWLEDGMENTS}

This work was supported by the U.S. Navy Office of Naval Research Ocean Acoustics Program and Boston University. The authors acknowledge the efforts of Eun-Joo Park, who carried out the bubble size measurements and analysis presented in Sec. V.

${ }^{1}$ T. G. Leighton, The Acoustic Bubble (Academic, London, 1994).

${ }^{2}$ H. Medwin and C. S. Clay, Fundamentals of Acoustical Oceanography (Academic, Boston, 1998).

${ }^{3}$ K. W. Commander and A. Prosperetti, "Linear pressure waves in bubbly liquids: Comparison between theory and experiments," J. Acoust. Soc. Am. 85, 732-746 (1989).
${ }^{4}$ E. Silberman, "Sound velocity and attenuation in bubbly mixtures measured in standing wave tubes," J. Acoust. Soc. Am. 29, 925-933 (1957).

${ }^{5} \mathrm{Z}$. Ye and L. Ding, "Acoustic dispersion and attenuation relations in bubbly mixture," J. Acoust. Soc. Am. 98, 1629-1636 (1995).

${ }^{6} \mathrm{C}$. Feuillade, "The attenuation and dispersion of sound in water containing multiply interacting air bubbles," J. Acoust. Soc. Am. 99, 3412-3430 (1996).

${ }^{7}$ F. S. Henyey, "Corrections to Foldy's effective medium theory for propagation in bubble clouds and other collections of very small scatters," J. Acoust. Soc. Am. 105, 2149-2154 (1999).

${ }^{8}$ S. G. Kargl, "Effective medium approach to linear acoustics in bubbly liquids," J. Acoust. Soc. Am. 111, 168-173 (2002).

${ }^{9}$ S. A. Cheyne, C. T. Stebbings, and R. A. Roy, "Phase velocity measurements in bubbly liquids using a fiber optic laser interferometer," J. Acoust. Soc. Am. 97, 1621-1624 (1995).

${ }^{10}$ P. S. Wilson, R. A. Roy, and W. M. Carey, “An improved water-filled impedance tube," J. Acoust. Soc. Am. 113, 3245-3252 (2003).

${ }^{11} \mathrm{~V}$. Gibiat and F. Laloë, "Acoustical impedance measurements by the twomicrophone-three-calibration (TMTC) method," J. Acoust. Soc. Am. 88, 2533-2545 (1990).

${ }^{12} \mathrm{P}$. S. Wilson, "Sound propagation in bubbly liquids," Ph.D. dissertation, Boston University, 2002.

${ }^{13}$ L. D. Lafleur and F. D. Shields, "Low-frequency propagation modes in a liquid-filled elastic tube waveguide," J. Acoust. Soc. Am. 97, 1435-1445 (1995).

${ }^{14}$ A. Jillavenkatesa, S. J. Dapkunas, and L.-S. H. Lum, Particle Size Characterization, U.S. Government Printing Office, Washington DC, 2001.

${ }^{15}$ A. B. Wood, A Textbook of Sound, 2nd ed. (G. Bell and Sons, London, 1941).

${ }^{16}$ M. Nicholas, R. A. Roy, L. A. Crum, H. Og̃uz, and A. Prosperetti, “Sound emissions by a laboratory bubble cloud," J. Acoust. Soc. Am. 95, 31713182 (1994).

${ }^{17}$ J. S. Bendat and A. G. Piersol, Random Data: Analysis and Measurement Procedures (Wiley-Interscience, New York, 1971).

${ }^{18}$ J. A. Schindall, “Acoustic scattering from compact bubble clouds," Ph.D. dissertation, The University of Mississippi, 1995.

${ }^{19}$ J. S. Bendat and A. G. Piersol, Engineering Applications of Correlation and Spectral Analysis, 2nd ed. (Wiley, New York, 1993). 